\title{
Molecular Modeling of Potential Dual Inhibitors of HIV Reverse Transcriptase and Integrase
}

\author{
Alberto Cabrera1, Leonor Huerta Hernández², Daniel Chávez³, \\ José L. Medina-Franco ${ }^{*}$ \\ ${ }^{1}$ Departamento de Farmacia, Facultad de Química, Universidad Nacional Autónoma de México, Mexico City, Mexico \\ ${ }^{2}$ Instituto de Investigaciones Biomedicas, Universidad Nacional Autónoma de México, Mexico City, Mexico \\ ${ }^{3}$ Centro de Graduados e Investigaciónen Químicadel InstitutoTecnológico de Tijuana, Tijuana, B.C., Mexico \\ Email: ^medinajl@unam.mx, jose.medina.franco@gmail.com
}

How to cite this paper: Cabrera, A., Hernández, L.H., Chávez, D. and Medina-Franco, J.L. (2018) Molecular Modeling of Potential Dual Inhibitors of HIV Reverse Transcriptase and Integrase. Computational Molecular Bioscience, 8, 1-41.

https://doi.org/10.4236/cmb.2018.81001

Received: February 15, 2018

Accepted: March 16, 2018

Published: March 19, 2018

Copyright $\odot 2018$ by authors and Scientific Research Publishing Inc. This work is licensed under the Creative Commons Attribution International License (CC BY 4.0).

http://creativecommons.org/licenses/by/4.0/

\begin{abstract}
With the goal of suggesting dual inhibitors of HIV reverse transcriptase (RT) and integrase (IN), herein we report the molecular docking of an initial set of 556 compounds related to the pyridinone class. Docking with multiple crystallographic structures of HIV-1 RT led to 160 potential binders of RT interacting with key amino acid residues at the enzyme's allosteric site. Compounds selected from the docking with RT were further docked with a crystallographic structure of HIV-1 IN. A total of 31 structures had the potential to make contacts with $\mathrm{Mg}^{2+}$ ions located in a small space between DNA and IN. Interactions with $\mathrm{Mg}^{2+}$ ions are relevant because they participate in the stabilization of the IN-DNA complex. In conclusion, 31 compounds synthetically accessible are proposed as dual inhibitors of RT and IN. It is hypothesized that the suggested compounds will inhibit RT by occupying the allosteric site for NNRTIs and will inhibit the catalytic activity of IN by destabilizing the IN-DNA complex. The main perspective of this work is the synthesis and biological testing of the candidate molecules.
\end{abstract}

\section{Keywords}

AIDS, Antiviral, Computer-Aided Drug Design, Docking, Polypharmacology

\section{Introduction}

Acquired Immune Deficiency Syndrome (AIDS) continues to be a major health problem in the world. In 2016, there were 1.0 million AIDS-related deaths and 36.7 million people living with the human immunodeficiency virus (HIV) [1]. 
Several compounds have been developed for the treatment of patients infected with HIV-1 [2]-[7]. These compounds, based on the mechanism of action, can be classified into five major groups: CCR5 blockers, fusion inhibitors, reverse transcriptase (RT) inhibitors (that include nucleoside, NRTIs and nonnucleoside, NNRTIs), integrase (IN), and protease (PI) inhibitors [8]. The molecular targets are involved in different aspects of the HIV virus. For instance, RT is responsible for producing proviral DNA from viral RNA, and IN is responsible for taking the proviral DNA and introducing it to cellular DNA in the nucleus [8] [9]. Figure 1 shows examples of compounds that the Food and Drug Administration (FDA) of the United States has approved for clinical use for the treatment of AIDS. The figure also shows examples of different chemical classes under development. Examples of NNRTI are Nevirapine, Efavirenz, Delavirdine, Etravirine, Rilpivirine, UC-781, HEPT, TNK 651, pyridin-2(1H)-one-UC781 hybrid, pyridin-2(1H)-one DH-10, and quinol-2(1H)-one DA-3 [10]. Examples of IN inhibitors are Elvitegralvir, Raltegravir, S-1360, and L-870810 (Figure 1).

HIV infection is currently controlled through combinations of drugs described above, collectively known as the Highly Active Antiretroviral Therapy (HAART). In this polypharmacy approach [11], the FDA has approved for clinical use multiclass combination products that contain PI or NRTI combined
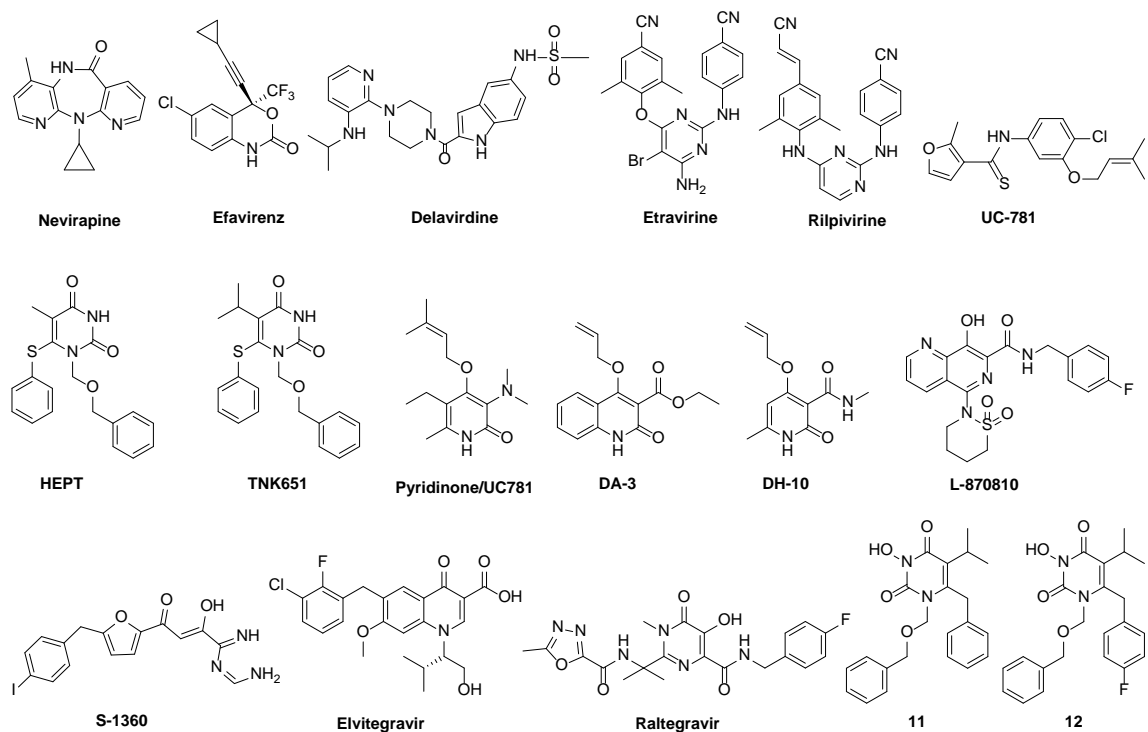

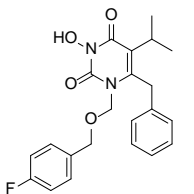

13

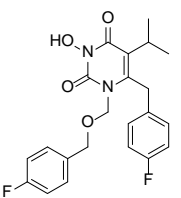

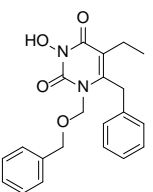

15

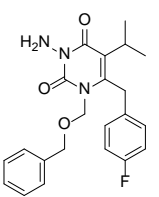

16

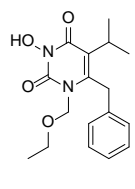

17

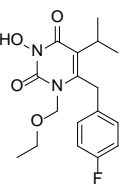

18

$$
\text { Wang et } a=11,12,13,14
$$

Figure 1. Representative compounds discussed in this work. Drugs approved for the treatment of HIV AIDS and other compounds under development. 
with NNRTI or IN inhibitors. One example is Atripla ${ }^{\star}$ that is a combination of three compounds, including the NNRTI Efavirenz. Other examples are Comple$\mathrm{ra}^{\circledR}$ and Stribild ${ }^{\oplus}$ which contains Rilpivirine and Elvitegravir, respectively [12]. On the other hand, a polypharmacology approach [11], attempts to develop dual active compounds such as 11 - 18 (Figure 1) that inhibit both RT and IN [6]. Since the induction of adverse side effects and the emergence of drug-resistant strains of HIV are major challenges of anti-HIV therapies, the development of multi-target drugs is an alternative to increase antiviral activity and to reduce the number of components in the combinations currently used [11].

Pyridinone derivatives are promising NNRTIs. Merck first reported the development of pyridinone derivatives as NNRTIs identifying highly potent molecules. However, investigation on this class of compounds was later stopped due to the induction of resistant mutant strains. However, in the past few years, other academic research groups have continued developing pyridinone derivatives leading to compounds with improved activity profile versus mutant strains of HIV-1 [5] [13] [14] [15]. Amongst these compounds are pyrimidinediones [16], which contain an $\mathrm{OH}$ group in $\mathrm{N}-1$ of the pyridione ring and that were proposed as dual inhibitors of RT and IN (Figure 1) [6].

The goal of this work was to assess the potential ability of pyridinone analogues and related compounds to act as dual inhibitors of RT and IN. The rationale is that dual inhibitors may be more effective than molecules directed to only one molecular target [17]. The rationale is that the proposed pyridinone derivatives (cf. Figure 2) are structurally related to the 3-hydroxypyrimidine-2,4-diones that are dual inhibitors of RT and IN as showed by Wang et al. All the compounds evaluated in this study (cf. Figure 2) are synthetically accessible. As discussed in section 2, the designed structures have features of the pyridinone-UC781 hybrid, that might maintain activity against mutant strains [4]. In<smiles>[R]c1c([R])c2ccccc2[nH]c1=O</smiles>

$\mathrm{OH}$<smiles>[R3]c1c([R])c2cc(Br)ccc2[nH]c1=O</smiles>

$\mathrm{OH}$<smiles>[R]c1c([R])c2cc(Br)ccc2[nH]c1=O</smiles><smiles>[R3]c1c([R3])c2cc(Cl)ccc2n(O)c1=O</smiles><smiles></smiles><smiles>[R3]c1c([R3])c2cc(I)ccc2[nH]c1=O</smiles><smiles>CC(=O)c1[nH]c2ccc(I)cc2c1Br</smiles><smiles></smiles><smiles></smiles>
OH<smiles></smiles>
$\mathrm{OH}$<smiles>[R3]c1c2c(n(O)c(=O)c1[R])CCC2</smiles>
$\mathrm{OH}$<smiles></smiles><smiles>[R]c1c(Br)[c][nH]c(=O)c1Br</smiles><smiles>CCC1C(C)=C(C)C(Br)=C1C</smiles>

Figure 2. Chemical structures of the pyrdinone derivatives considered in this work. 
particular, the new structures could preserve the activity against Tyr181Cys mutant strains because of the flexibility of the side chain at C-4 (substituent R4 in Figure 2) [4].

\section{Methods}

Based on the structure of the pyridinone-UC781 hybrid proposed earlier [4], chemical structure of 556 compounds was initially proposed based on synthetic accessibility (Figure 2). The overall rationale of the design was to introduce a polar group at C-3 and an unsaturated aliphatic chain in C-4. The chemical structures of the 3-hydroxypyrimidine-2,4-diones developed by Wang et al. were also considered in the design. In particular, the introduction of an N-OH substitution would lead to candidate compounds able to act as dual inhibitors, inhibiting both RT and IN [6].

The crystallographic structures of the biomolecular targets were retrieved from the Protein Data Bank (PDB) (www.rcsb.org) [18]. Table 1 summarizes the information of the three structures of RT [15] and one for IN [19] [20] used in this work, including the information of the co-crystallized ligand. All computational studies were conducted with Molecular Operating Environment (MOE) software, version 2014 [20].

Table 1. Summary of the crystallographic structures of RT and IN used in this work [15] [19].

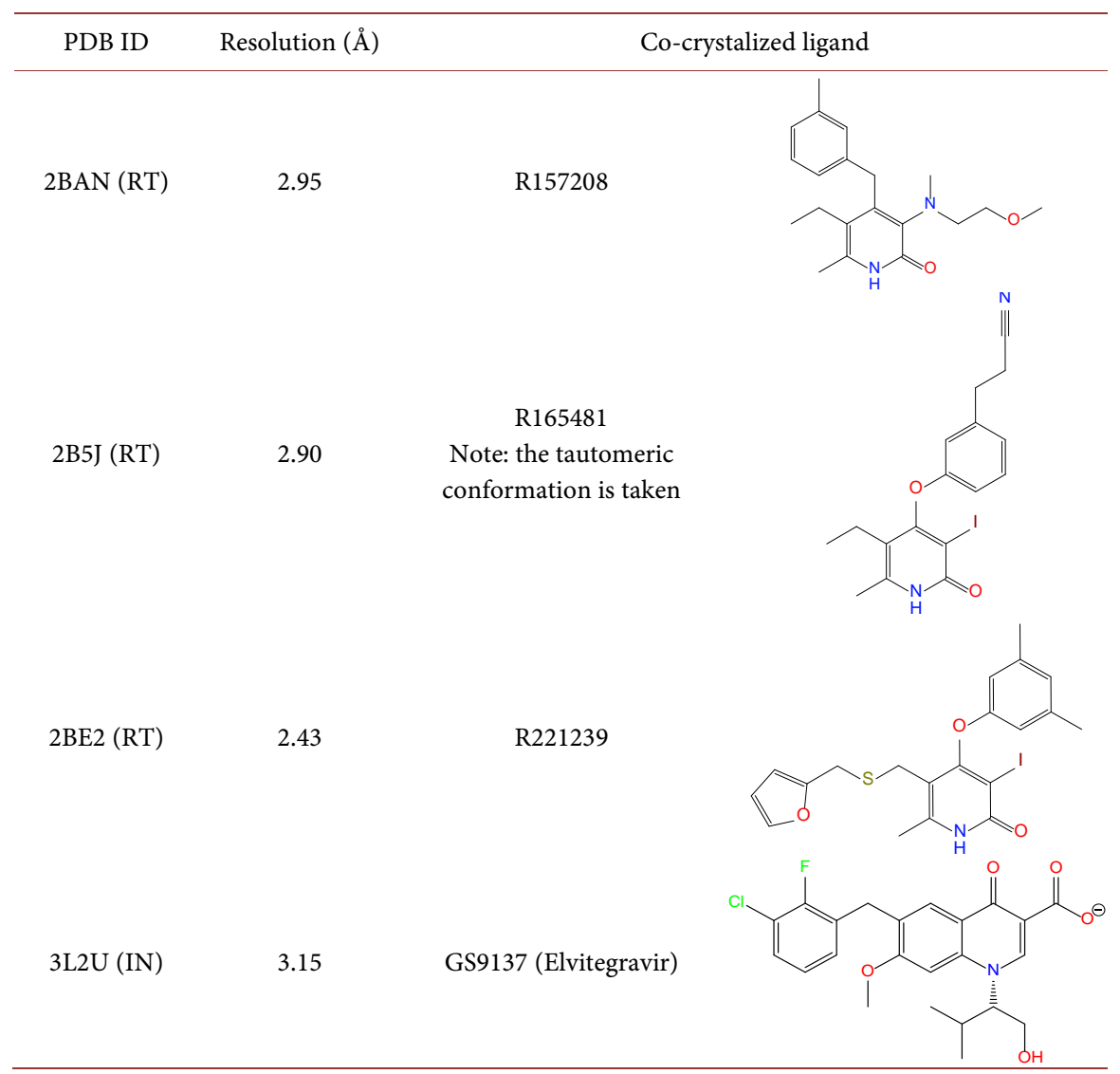




\subsection{Structure Preparation}

\subsubsection{Reverse Transcriptase}

In each of the three crystallographic structures of RT (Table 1) manganese ions, glycerol, sucrose, and water molecules (except HOH1013 in PDB ID: 2B5J) were eliminated. Then the geometry of the structures was optimized with the $\operatorname{LigX}$ module available in MOE using default settings. Before docking, the coordinates of the three crystallographic structures of RT were aligned using the chain A as template.

\subsubsection{Integrase}

For IN, the crystallographic structure of foamy virus (PFV) was taking as a starting point similar to the work of Wang et al. [6] [17]. As in the preparation of the structure of RT, non-amino acid ligands were removed from the structure except Elvitegravir (GS9137, Table 1) i.e., zinc ions, glycerol and ammonia molecule. The geometry of the structure was optimized with the LigX module available in MOE using default settings.

\subsection{Validation of Docking Protocol}

Before docking the new compounds in Figure 2, the docking protocol was validated by re-docking the co-crystal ligands in their corresponding crystallographic structure (Table 1). During docking, the structure of the co-crystal ligands (i.e., R221239, R165481, R157208 and GS9137) was considered semi-flexible. The docking was done with the MMFF94x force field using default options of MOE (500 iterations in total with 30 consecutive attempts to select the best result). The binding pocket was defined as the set of amino acids within of $4.5 \AA$ of the co-crystal ligand.

\subsection{D Flexible Alignment of Pyridinone Structures}

In order to explore if the compounds in Figure 2 could adopt a similar conformation as the co-crystalized pyridinone analogues, a representative set of 56 (10\%) molecules were aligned flexibly to the co-crystal coordinates of R221239, R165481, and R157208 (Table 1). The chemical structures of the 56 selected compounds are in the Supplementary material. During the alignment, the structure of the co-crystal compound was kept rigid. The flexible alignment conducted in MOE was done using default settings (500 iterations in total with 30 consecutive attempts to find the best result) with the MMFF94x force field.

\subsection{Docking}

\subsubsection{Docking with RT}

All 556 compounds were docked with the crystallographic structures PDB ID: 2BAN and 2B5J using the same settings of the validation step. After docking with the two crystallographic structures, 160 compounds were selected for further analysis. As part of the analysis, in particular the binding poses, protein ligand interaction fingerprints (PLIFs) were generated with MOE. Of note, the 
new compounds in Figure 2 were not docked with PDB ID: 2BE2 based on the results of the flexible alignment detailed in Section 2.3 i.e., the proposed compounds did not adopted a similar conformation to R221239 (vide infra).

\subsubsection{Docking with IN}

160 compounds selected from the docking with RT (Section 2.4.1) were docked with the crystallographic structure PDB ID: 3L2U using the same parameters used in the docking of the co-crystal ligand (Elvitegravir, GS9137) [19] during the validation step. As discussed on the sequel, 76 pyridinone analogues structures were selected based on the binding poses and resemblance of the functional groups of reported dual inhibitors of RT and IN [17].

\subsection{Calculation of Drug-Like Properties}

In order to assess the potential oral bioavailability of the newly proposed compounds, we calculated properties of pharmaceutical interest, namely [21] [22]; molecular weight (MW), the partition coefficient octanol/water (Log P) as a measure of lipophilicity, topological polar surface area (TPSA), number of hydrogen bond donors (HBD), hydrogen bond acceptors (HBA), and rotatable bonds (RB).

\section{Results and Discussion}

\subsection{Alignment of Crystallographic Structures of RT}

The three crystallographic structures of RT (Table 1) were aligned before the docking. Figure S1 in the Supplementary material summarizes the results of the alignment. Results of the alignment indicated that, overall, the conformation of the crystallographic structures is similar (RMSD values between $0.77 \AA$ and 1.23 $\AA$ ). Analysis of the conformation of the side chains in the binding pocket revealed important differences in the side chain of Tyr181 in PDB ID: 2BAN. The different position of this side chain is due to the bulky substituent at C-5 of the pyridinone ring of R221239, as compared to the small (ethyl) substituent at the same C-5 position of R157208 and R165481 in PDB ID: 2BAN and 2B5J, respectively. The coordinates of the aligned and superposed structures were used for docking of 556 pyridinones.

\subsection{Validation of the Docking Protocol with RT}

Docking of the co-crystal ligands with their corresponding crystallographic structures yield excellent results with low $(<1 \AA$ ) RMSD values: 0.8832, 0.8925 and 0.7379 for 2BAN, 2BE2 and 2B5J, respectively. Figure S2 in the Supplementary material illustrates the results of the validation. These results indicated that the settings used in MOE were able to reproduce the binding modes observed in the crystal structure. The relative docking scores of the co-crystal ligands with the corresponding structure of RT were $-9.00,-1.01$, and -8.63 for $2 \mathrm{BAN}, 2 \mathrm{BE} 2$ and $2 \mathrm{~B} 5 \mathrm{~J}$, respectively. 


\subsection{Alignment with Co-Crystalized Pyridinone Derivatives}

As described in the Methods section, $56(10 \%)$ pyridinones were taken from the entire set of 556 molecules using a stratified random sampling strategy (Figure S6 in the Supplementary material). The selected structures were aligned flexibly with the co-crystallographic positions of R157208, R165481 and R221239 (Table 1). Table 2 summarizes the results of the $3 \mathrm{D}$ alignment including the scores. This value quantifies the quality of the alignment taking into account the $3 \mathrm{D}$ similarity of the molecules considering the average energy penalty (in $\mathrm{kcal} / \mathrm{mol}$ ) for the conformational restriction of the ligands associated with the alignment. Lower values (more negative) indicate a better alignment. The colors of the alignment values in Table 2 classify the relative magnitude of the alignment scores as compared to the average plus two standard deviations of the scores vs. template compounds. Values in green indicate highly favorable scores (i.e., better than the average plus two standard deviations), values in blue denote average values, and values in red indicate the less favorable scores (two standard deviations below average). Based on the average alignment scores the best alignments were obtained, in general, using R165481 as reference. Overall, R157208 was the template pyridinone with the second best alignment scores. Of note, R165481 and R157208 have a small (ethyl) substituent at C-5. In contrast, R221239 (with a bulky substituent at C-5) led to less favorable alignment scores. These results are in agreement with the structures of the new compounds considered in this work (vide supra) (Figure 2).

Figure 3 shows the results of 3D-alignments of representative compounds, 1 and 77 with R157208 and R165481, respectively. In the figure is possible to observe that, in general, the structures of 1 and 77 have a good overlap with the reference molecules.

The conformation of the aligned compounds was overlapped in the crystallographic structure of RT (Figure 4). Note that despite the fact that the quinolone ring of 1 aligned with R165481 is flipped by about $180^{\circ}$ vs. the pyridnone ring of the template compound, it is capable of occupy the allosteric site of RT. In the alignment-based conformation shown in Figure 4, 1 could interact with Leu100 and Lys101 making a hydrogen bond with Lys101. The same hydrogen bond could be formed with the tautomeric structure R165481 (Figure 4). The hydrogen bond interaction with Lys101 is present in other NNRTIs structurally similar to pyridinone like pyrimidines [23].

The binding poses of the 556 pyridinones docked with two crystallographic structures of RT (PDB IDs: 2BAN and 2B5J) showed a hydrogen bond interaction between the amine of the pyridinone ring with the oxygen atom of the carbonyl group of Lys101. This hydrogen bond is observed for several NNRTIs including R157208 and R165481 [4] [24] [25]. In order to analyze the results, we selected the compounds that had predicted contacts with Tyr181 and Tyr188, and structures that interact with conserved amino acids Trp229, Pro236 and Tyr3118. To support this analysis we used the results of PLIFs fully detailed in 
Table 2. 3D alignment scores $(\mathrm{kcal} / \mathrm{mol})$ calculated with MOE of 56 selected compound with the structure of three co-crystallized pyridinones. ${ }^{*}$ The structure of each compound is shown in the Supplementary material.

\begin{tabular}{|c|c|c|c|c|c|}
\hline ID & R157208 & ID & R221239 & ID & R165481 \\
\hline 207 & -95.19 & 487 & -100.47 & 77 & -98.46 \\
\hline 357 & -90.35 & 317 & -94.82 & 1 & -97.37 \\
\hline 117 & -89.12 & 77 & -89.94 & 277 & -95.92 \\
\hline 497 & -88.70 & 367 & -87.64 & 61 & -92.80 \\
\hline 467 & -86.36 & 61 & -87.51 & 317 & -92.74 \\
\hline 337 & -85.38 & 337 & -86.93 & 127 & -92.64 \\
\hline 147 & -84.98 & 51 & -86.15 & 537 & -92.34 \\
\hline 287 & -84.36 & 447 & -85.25 & 377 & -92.28 \\
\hline 547 & -84.17 & 21 & -84.63 & 41 & -91.84 \\
\hline 1 & -83.94 & 237 & -84.58 & 327 & -91.53 \\
\hline 377 & -83.42 & 397 & -83.87 & 517 & -90.80 \\
\hline 97 & -83.01 & 117 & -83.81 & 367 & -90.28 \\
\hline 477 & -82.84 & 137 & -83.59 & 287 & -89.89 \\
\hline 247 & -81.54 & 307 & -81.51 & 31 & -89.42 \\
\hline 107 & -79.99 & 457 & -80.16 & 257 & -89.25 \\
\hline 137 & -79.68 & 1 & -79.05 & 547 & -89.15 \\
\hline 61 & -79.64 & 537 & -78.27 & 71 & -87.75 \\
\hline 487 & -79.09 & 11 & -78.05 & 137 & -86.29 \\
\hline 77 & -78.81 & 277 & -77.25 & 417 & -86.20 \\
\hline 417 & -77.85 & 41 & -76.94 & 11 & -85.61 \\
\hline 197 & -77.72 & 127 & -76.67 & 497 & -84.72 \\
\hline 127 & -76.25 & 527 & -76.52 & 487 & -84.63 \\
\hline 157 & -75.33 & 207 & -74.99 & 87 & -84.41 \\
\hline 227 & -74.71 & 147 & -74.56 & 107 & -84.34 \\
\hline 277 & -74.24 & 167 & -73.48 & 527 & -83.70 \\
\hline 51 & -74.17 & 377 & -73.32 & 207 & -83.39 \\
\hline 447 & -73.85 & 517 & -73.17 & 337 & -83.08 \\
\hline 267 & -73.28 & 71 & -72.74 & 467 & -82.95 \\
\hline 427 & -73.16 & 257 & -72.58 & 147 & -82.89 \\
\hline 41 & -72.63 & 477 & -71.98 & 397 & -82.66 \\
\hline 457 & -71.99 & 287 & -71.85 & 447 & -82.17 \\
\hline 527 & -71.53 & 407 & -71.80 & 407 & -81.48 \\
\hline 307 & -71.46 & 227 & -71.23 & 167 & -81.34 \\
\hline 87 & -71.07 & 87 & -71.21 & 307 & -80.04 \\
\hline 437 & -70.95 & 217 & -70.86 & 51 & -79.58 \\
\hline
\end{tabular}




\section{Continued}

\begin{tabular}{|c|c|c|c|c|c|}
\hline 407 & -70.19 & 437 & -68.79 & 457 & -78.90 \\
\hline 167 & -69.39 & 497 & -67.48 & 157 & -78.80 \\
\hline 517 & -69.34 & 31 & -66.88 & 477 & -78.01 \\
\hline 537 & -69.33 & 417 & -66.72 & 117 & -76.66 \\
\hline 327 & -69.13 & 347 & -65.84 & 97 & -76.10 \\
\hline 71 & -68.89 & 157 & -65.05 & 507 & -75.50 \\
\hline 11 & -68.81 & 187 & -65.03 & 347 & -74.90 \\
\hline 317 & -67.16 & 467 & -64.97 & 177 & -74.64 \\
\hline 297 & -66.27 & 297 & -62.65 & 267 & -73.91 \\
\hline 237 & -66.12 & 507 & -59.88 & 387 & -73.00 \\
\hline 257 & -65.11 & 247 & -59.46 & 237 & -72.54 \\
\hline 177 & -65.03 & 267 & -59.31 & 21 & -71.72 \\
\hline 21 & -64.90 & 197 & -56.36 & 357 & -71.47 \\
\hline 507 & -61.96 & 547 & -56.26 & 187 & -68.96 \\
\hline 367 & -61.52 & 97 & -55.40 & 217 & -68.66 \\
\hline 187 & -61.09 & 357 & -55.04 & 437 & -68.12 \\
\hline 397 & -60.77 & 177 & -52.55 & 197 & -66.33 \\
\hline 217 & -58.21 & 327 & -52.00 & 297 & -65.39 \\
\hline 387 & -56.81 & 427 & -48.64 & 227 & -64.74 \\
\hline 31 & -56.10 & 387 & -48.41 & 247 & -62.67 \\
\hline 347 & -54.72 & 107 & -46.84 & 427 & -60.66 \\
\hline Average & -73.38 & Average & -71.98 & Average & -81.53 \\
\hline St. dev. & \pm 9.39 & St. deviation & \pm 12.25 & St. deviation & \pm 9.36 \\
\hline
\end{tabular}

*Based on the average and the standard deviation of the calculated energies, values in green denote outstanding results (more negative), values in blue are close to the average, and values in red are below (less negative) the average.

the Supplementary material (Figure S5). Table 3 and Table 4 summarize the docking scores of the selected compounds with two crystal structures of RT. The more negative values indicate more favorable docking. Table 3 shows the results with PDB ID: 2BAN and Table 4 with PDB ID: 2B5J. In both tables, compounds in the left column are the ones able to make contacts with Tyr181-Tyr188 and those on the right column make contact with Trp-Pro236 or Trp229-Pro236-Tyr318, respectively.

\subsection{Docking with RT}

Based on the results of the $3 \mathrm{D}$ flexible alignment discussed in section 3.3, the structure PDB ID: 2BE2 structure was no longer considered for docking analysis. As discussed, this was because the results suggested that the geometry of the binding site of R221239 bound to RT is not representative for the group of compounds studied in this work. 
1
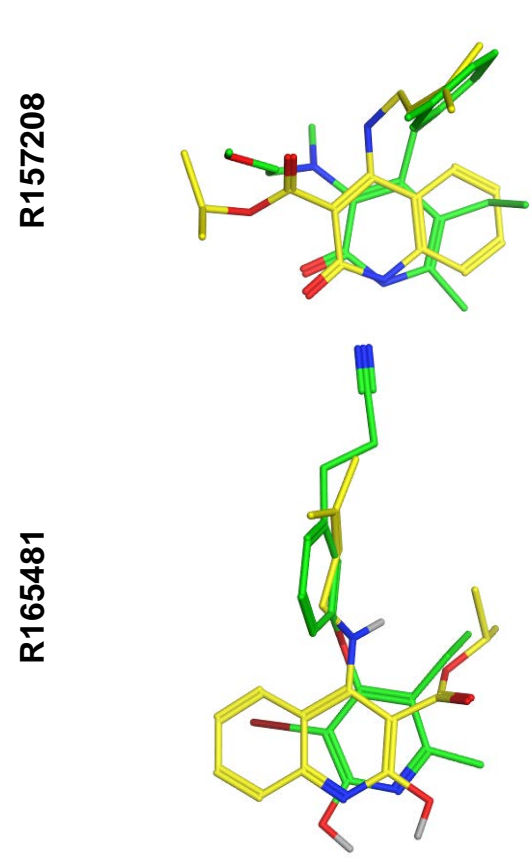

77
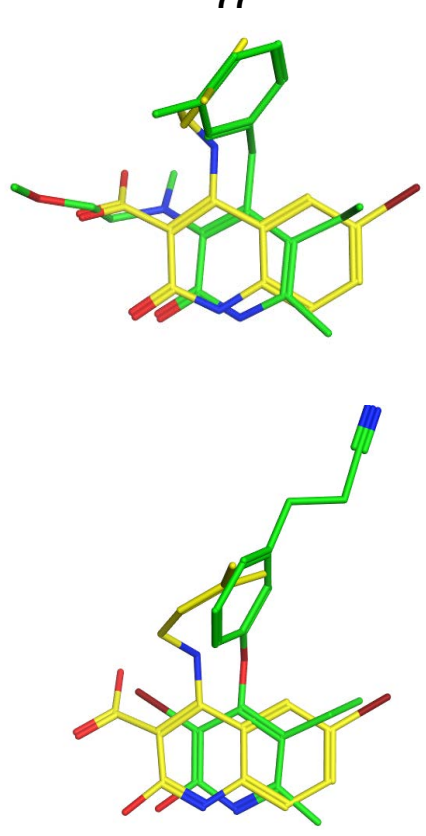

Figure 3. Flexible alignment of representative compounds 1 and 77 (carbon atoms in yellow) with the co-crystal coordinates of R157208 and R165481 (carbon atoms in green). The alignment scores are in Table 2.
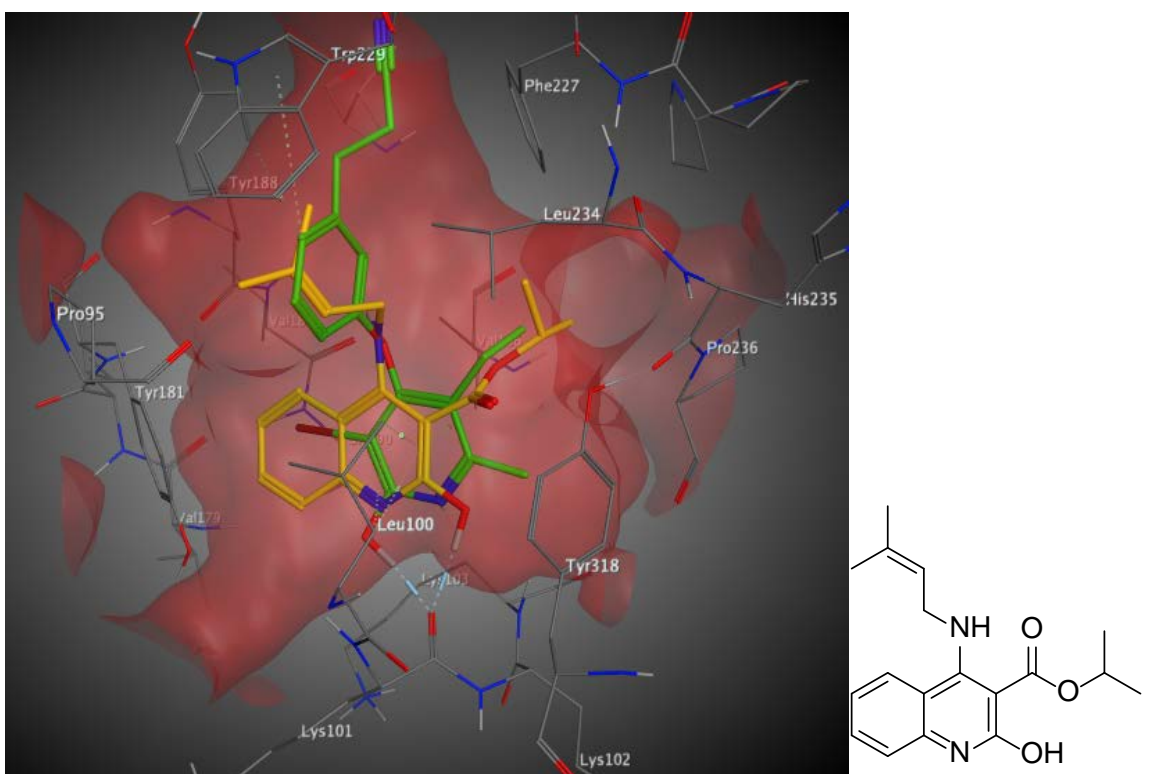

Figure 4. Alignment-based conformation of 1 (quinolone) with R165481 (atoms in green color) inside the binding pocket of PDB ID: 2B5J. This conformation shows an interaction with Leu100; and, Lys101 with a hydrogen bond.

\subsubsection{Binding Modes with RT, PDB ID: 2BAN}

Based on the docking scores and predicted contacts with key amino acids (Table 3 , values in green font), 13 compounds with the best docking profile were selected. Six compounds showed favorable docking scores and interactions with 
Table 3. Docking results with RT PDB ID: 2BAN. The cells are colored to highlight major amino acids involved in protein-ligand interactions. Gray and yellow color indicate interaction with Tyr181 and Tyr188, respectively. Cells in blue and green denote interactions with Pro236 and Trp229, respectively. Considering the average and the standard deviation of the docking scores, values in green font indicate the most favorable results, values in blue font indicate average scores, and values in red font denote the less favored scores.

\begin{tabular}{|c|c|c|c|}
\hline ID & Tyr181-188 (2BAN) & ID & Trp229-Pro236 (2BAN) \\
\hline 211 & -8.38 & 231 & -7.69 \\
\hline 146 & -8.29 & 153 & -7.60 \\
\hline 446 & -8.09 & 72 & -7.44 \\
\hline 226 & -8.05 & 74 & -7.31 \\
\hline 101 & -7.96 & 178 & -7.28 \\
\hline 114 & -7.94 & 162 & -7.23 \\
\hline 538 & -7.81 & 73 & -7.20 \\
\hline 287 & -7.77 & 102 & -7.07 \\
\hline 250 & -7.76 & 551 & -7.03 \\
\hline 206 & -7.69 & 35 & -7.03 \\
\hline 189 & -7.67 & 34 & -7.02 \\
\hline 192 & -7.66 & 311 & -7.01 \\
\hline 343 & -7.62 & 219 & -6.96 \\
\hline 100 & -7.58 & 299 & -6.88 \\
\hline 439 & -7.52 & 124 & -6.80 \\
\hline 354 & -7.47 & 169 & -6.74 \\
\hline 161 & -7.46 & 356 & -6.63 \\
\hline 338 & -7.41 & 259 & -6.63 \\
\hline 333 & -7.39 & 210 & -6.59 \\
\hline 74 & -7.31 & 308 & -6.56 \\
\hline 177 & -7.26 & 1 & -6.53 \\
\hline 447 & -7.26 & 526 & -6.52 \\
\hline 299 & -7.24 & 267 & -6.47 \\
\hline 156 & -7.12 & 271 & -6.33 \\
\hline 249 & -7.12 & 459 & -6.21 \\
\hline 113 & -7.05 & 402 & -6.19 \\
\hline 551 & -7.03 & 439 & -6.15 \\
\hline 237 & -6.97 & 38 & -6.11 \\
\hline 445 & -6.86 & 132 & -6.11 \\
\hline 193 & -6.86 & 133 & -6.10 \\
\hline 323 & -6.67 & 433 & -6.09 \\
\hline 364 & -6.63 & 362 & -6.01 \\
\hline
\end{tabular}




\section{Continued}

\begin{tabular}{|c|c|c|c|}
\hline 190 & -6.49 & 310 & -5.99 \\
\hline 173 & -6.44 & 332 & -5.92 \\
\hline 383 & -6.43 & 86 & -5.88 \\
\hline 429 & -6.28 & 525 & -5.76 \\
\hline 442 & -6.23 & 463 & -5.74 \\
\hline 97 & -6.22 & 252 & -5.61 \\
\hline 358 & -6.05 & 392 & -5.60 \\
\hline 268 & -6.04 & 447 & -5.53 \\
\hline 507 & -6.03 & 394 & -5.39 \\
\hline 11 & -5.97 & 79 & -5.32 \\
\hline 334 & -5.94 & 197 & -5.07 \\
\hline 233 & -5.32 & 423 & -3.94 \\
\hline Average & -7.10 & Average & -6.39 \\
\hline \multirow[t]{3}{*}{ Std. Dev. } & \pm 0.73 & Std. Dev. & \pm 0.75 \\
\hline & Tyr188 & & Pro236 \\
\hline & Tyr181 & & $\operatorname{Trp} 229$ \\
\hline
\end{tabular}

Tyr181 and Tyr188 (compounds 211, 146, 446, 226, 101, and 114). Seven compounds had favorable scores and interactions with Trp229 and Pro236 (231, 153, $72,74,178,162$ and 73). The chemical structures are shown in the Supplementary material (Figure S7) (PDF file "160 structures docked with RT"). Figure 5(a) and Figure 5(b) show the docking pose of selected compounds 211 and 231 with RT (PDB ID: 2BAN). Structures 211 and 231 had the best results for interactions with Tyr181 and the conserved amino acid Trp229, respectively. The structures of pyridinones 211 and 231 have characteristics similar to the pyridinone-UC781 hybrid (Figure 1) [4] [10]. Both binding models is predicted the characteristic hydrogen bond with Lys101. In addition, the substituent at C-4 (4-methylpent-3-en-1-oxyde) is flexible and can make contacts with Tyr181, Tyr188, Trp229, Pro236 or Tyr318. Such flexibility is important in mutant strains because the group in C-4 will help the compound to maintain the interaction with other amino acid in the allosteric site and in this way would favor activity against mutant RT [4].

Figure 6 illustrates a further example of this flexibility of the C-4 position (R4 substituent). In this binding model, compound 447 makes two hydrogen bond interactions with Lys101 and Lys103. At the same time, the molecule has the potential to interact with the side chains of Tyr 188 or Trp229 Through the substituent at C-4.

\subsubsection{Binding Modes with RT, PDB ID: 2B5J}

Table 4 summarizes the results of the docking with the structure of RT PDB ID: 2B5J. Out of the 556 docked compounds, 91 molecules had binding poses able to 
Table 4. Docking results with RT PDB ID: 2B5J. The cells are colored to highlight major amino acids involved in protein-ligand contacts. Gray and yellow color indicate interaction with Tyr181 and Tyr188, respectively. Cells in blue, green, and orange denote interactions with Pro236, Trp229, and Tyr318, respectively. Considering the average and the standard deviation of the results obtained, the values in red font indicate non-outstanding results (less negative). In blue, the acceptable results and in green are the outstanding results (more negative).

\begin{tabular}{|c|c|c|c|}
\hline ID & Tyr181-188 (2B5J) & ID & Trp229-Pro236-Tyr318 (2B5J) \\
\hline 315 & -8.09 & 450 & -8.83 \\
\hline 474 & -7.69 & 244 & -8.51 \\
\hline 224 & -7.62 & 486 & -8.50 \\
\hline 58 & -7.55 & 201 & -8.39 \\
\hline 310 & -7.52 & 487 & -8.30 \\
\hline 269 & -7.47 & 546 & -7.94 \\
\hline 470 & -7.44 & 448 & -7.71 \\
\hline 447 & -7.39 & 10 & -7.65 \\
\hline 299 & -7.38 & 513 & -7.59 \\
\hline 454 & -7.37 & 506 & -7.51 \\
\hline 236 & -7.35 & 535 & -7.45 \\
\hline 538 & -7.34 & 312 & -7.37 \\
\hline 94 & -7.27 & 288 & -7.33 \\
\hline 23 & -7.24 & 542 & -7.31 \\
\hline 59 & -7.22 & 445 & -7.25 \\
\hline 225 & -7.20 & 267 & -7.20 \\
\hline 550 & -7.19 & 540 & -7.18 \\
\hline 451 & -7.18 & 199 & -7.17 \\
\hline 227 & -7.17 & 531 & -7.17 \\
\hline 315 & -7.17 & 499 & -7.16 \\
\hline 529 & -7.15 & 299 & -7.14 \\
\hline 219 & -7.08 & 485 & -7.13 \\
\hline 309 & -7.04 & 305 & -7.11 \\
\hline 473 & -6.98 & 539 & -7.11 \\
\hline 151 & -6.94 & 44 & -7.07 \\
\hline 212 & -6.93 & 52 & -7.07 \\
\hline 235 & -6.89 & 505 & -7.06 \\
\hline 342 & -6.77 & 507 & -7.03 \\
\hline 49 & -6.68 & 120 & -6.88 \\
\hline 357 & -6.68 & 287 & -6.85 \\
\hline 515 & -6.65 & 54 & -6.84 \\
\hline 313 & -6.52 & 42 & -6.83 \\
\hline
\end{tabular}




\section{Continued}

\begin{tabular}{|c|c|c|c|}
\hline 69 & -6.48 & 538 & -6.80 \\
\hline 372 & -6.43 & 194 & -6.79 \\
\hline 472 & -6.37 & 529 & -6.75 \\
\hline 551 & -6.29 & 228 & -6.74 \\
\hline 296 & -6.26 & 550 & -6.70 \\
\hline 503 & -6.24 & 523 & -6.64 \\
\hline 554 & -6.20 & 18 & -6.64 \\
\hline 54 & -6.11 & 548 & -6.64 \\
\hline 16 & -5.95 & 41 & -6.47 \\
\hline 177 & -5.35 & 246 & -6.41 \\
\hline 513 & -5.17 & 496 & -6.39 \\
\hline 325 & -4.84 & 99 & -6.38 \\
\hline 340 & -4.30 & 503 & -6.34 \\
\hline 382 & -3.75 & 100 & -6.18 \\
\hline 95 & -2.35 & 333 & -6.14 \\
\hline Average & -6.64 & 328 & -6.02 \\
\hline \multirow[t]{11}{*}{ Std. Dev. } & \pm 1.08 & 555 & -5.82 \\
\hline & Tyr188 & 475 & -5.59 \\
\hline & Tyr181 & 541 & -5.11 \\
\hline & & 472 & -4.95 \\
\hline & & 340 & -4.30 \\
\hline & & 423 & -1.24 \\
\hline & & Average & -6.83 \\
\hline & & Std. Dev. & \pm 1.15 \\
\hline & & & Pro236 \\
\hline & & & $\operatorname{Trp} 229$ \\
\hline & & & Tyr318 \\
\hline
\end{tabular}

make interactions with Tyr181, Tyr188, Trp229, Pro236, or Tyr318. Ten compounds $(54,299,315,340,472,503,513,529,538$ and 550) can interact with other amino acid residues in the pocket. In all cases, compounds make a hydrogen bond interaction with Lys101.

For example, compound 315 had a favorable docking score and also was able to make hydrophobic contacts with Tyr181 (Table 4). Figure 7(a) and Figure 7 (b) show the two most favored binding poses for this molecule with docking scores of -8.09 and -7.17 , respectively. In both poses, it was observed the distinctive hydrogen bond interaction with Lys101. The results suggest the hypothesis that compound 315 may be active against RT.

Figure 8 depicts the binding model of 450 , a top ranked molecule (docking 

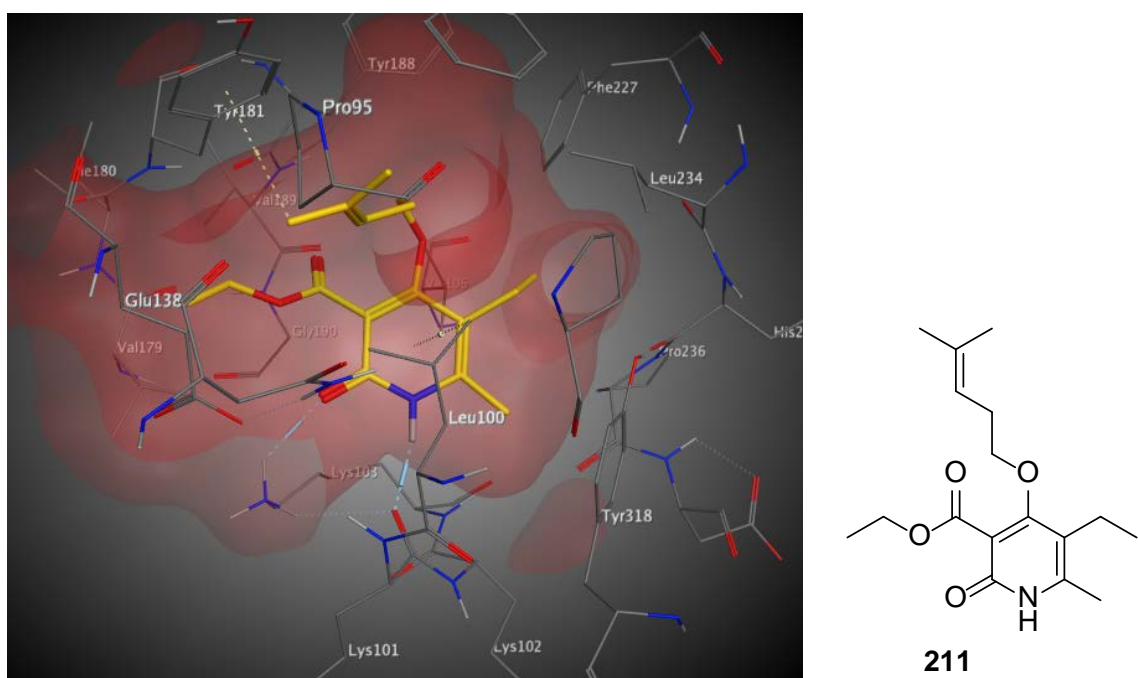

(a)

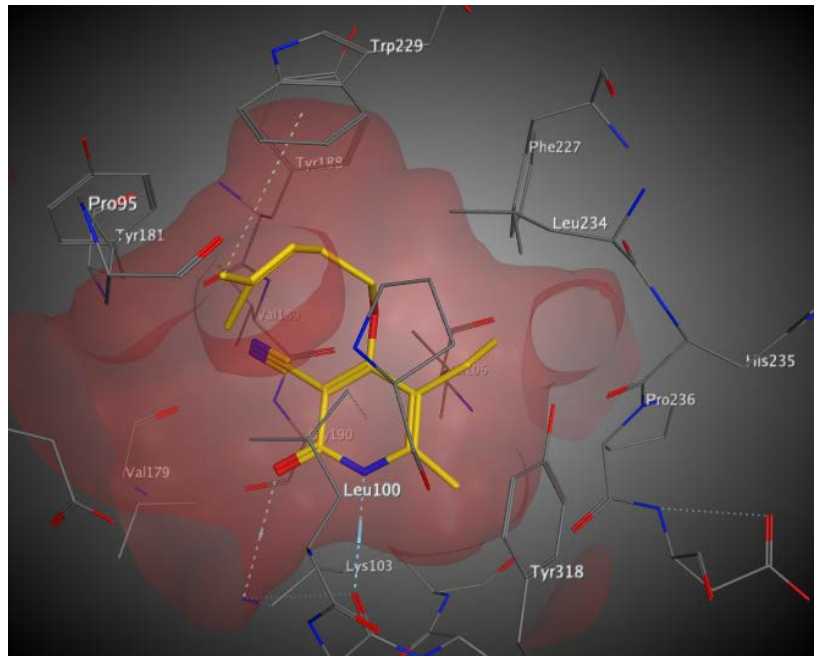

(b)

Figure 5. (a) Docking of structure 211 with RT (PDB ID: 2BAN). An interaction with the methyl group at C-4 and Tyr181; (b) Docking of structure 231 with RT (PDB ID: 2BAN). It is noteworthy the interaction of the methyl group of substituent in C-4 with Trp229.

score of -8.83) that is predicted to have contacts with Trp229 (Table 4). This compound can make hydrogen bond interactions with Lys101 and make contacts with Leu100. Figure 8 also depicts the binding model of 546 (docking score of -7.94). According to the binding model, 546 can make interactions with Trp229, Tyr188, and Tyr318. Because of the contacts with the side chains of the conserved amino acid Trp229, it is hypothesized that molecules such as 450 and 546 could be active against mutant strains of HIV-RT.

Figure 9 shows the binding model of 10 , a compound that is predicted to make contacts with Pro236. The docking model of compound 10 shows interactions with the ester substituent of C-3 and Pro236. Additional contacts are observed with Lys103 and Leu100, in addition of the hydrogen bond interaction with Lys101. 


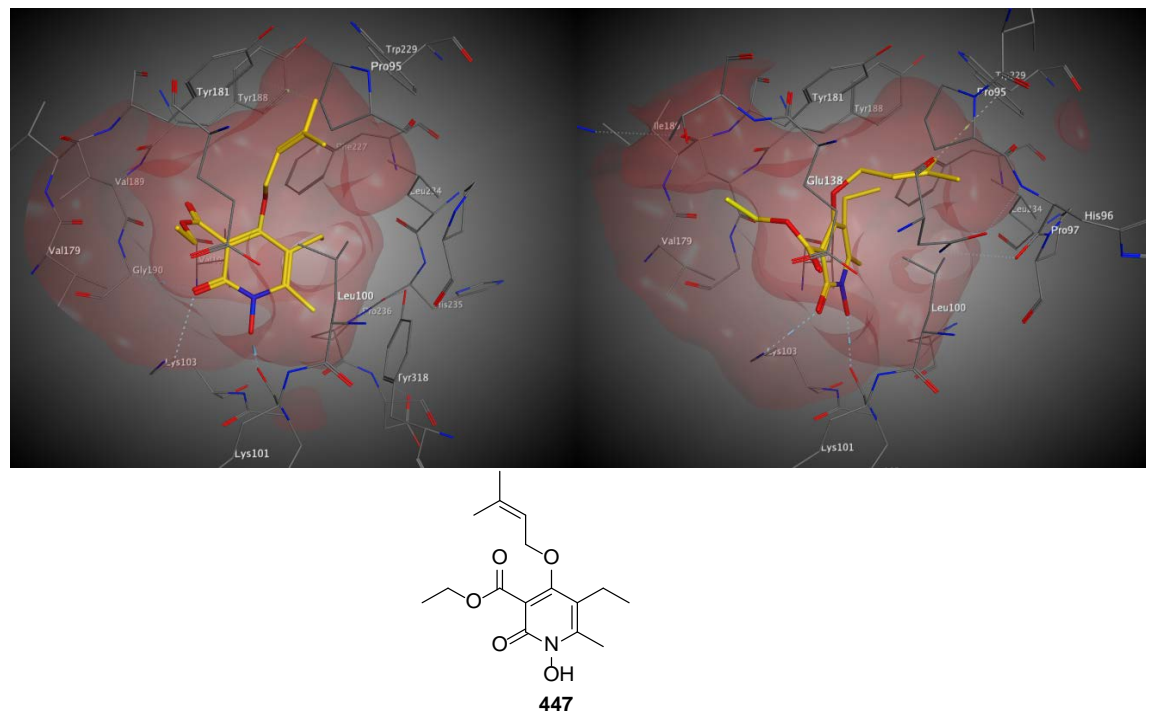

Figure 6. Possible docking poses of structure 447 with RT PDB: 2BAN. It is possible to observe the interaction of methyl group of substituent in C-4 with Tyr188 (a) and Trp229 (b) The hydrogen bond and the interaction with Lys103 is common to both poses.

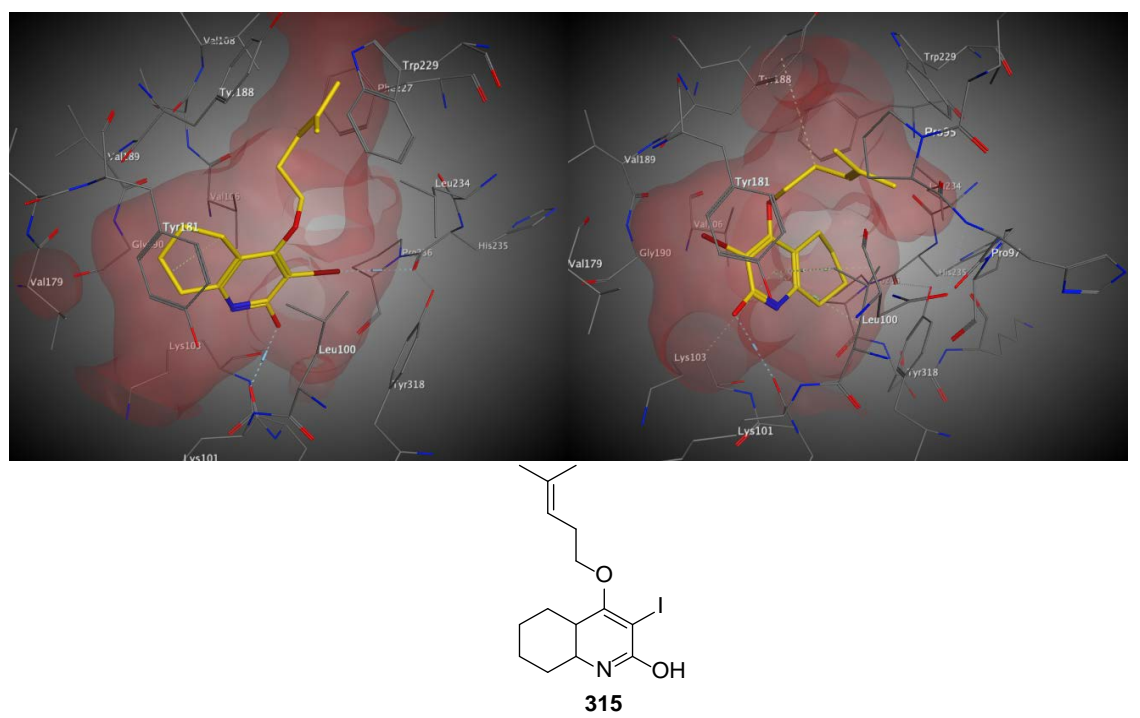

Figure 7. Docking of structure 315 with RT PDB: 2B5J where is possible to observe the interaction hydrogen of C-5 of cyclohexene and the aromatic portion of Tyr181 (a) and interaction is between a hydrogen of C-3' of substituent in C-4 with the aromatic portion of Tyr188 (b).

\subsection{Validation of the Docking Protocol with IN}

The docking protocol with IN was validated by re-docking the co-crystal ligand Elvitegravir. The RMSD value of $1.3 \AA$ indicated the ability of the docking protocol to reproduce the binding mode observed in the co-crystallized structure (see Figure S3 in the Supplementary material).

\subsection{Docking with IN}

A total of 76 compounds that had good docking results with RT (vide supra) 

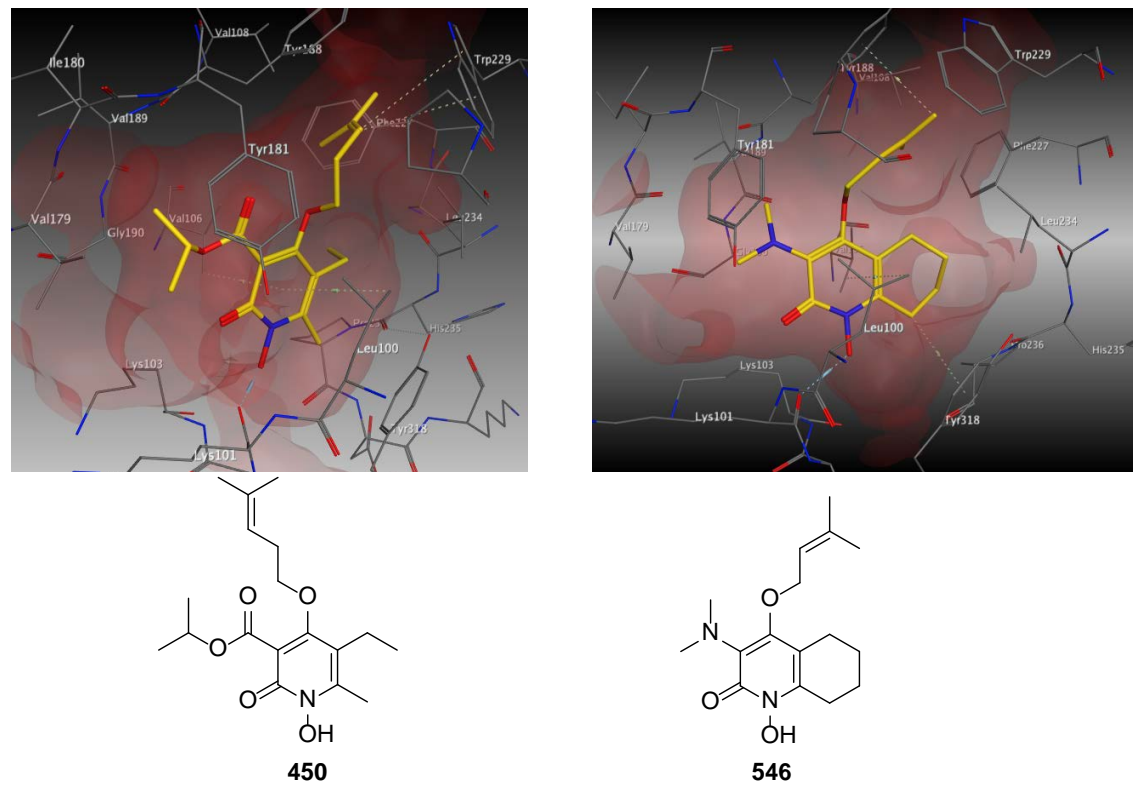

Figure 8. Docking of structure 450 and 546 with RT PDB: 2B5J.

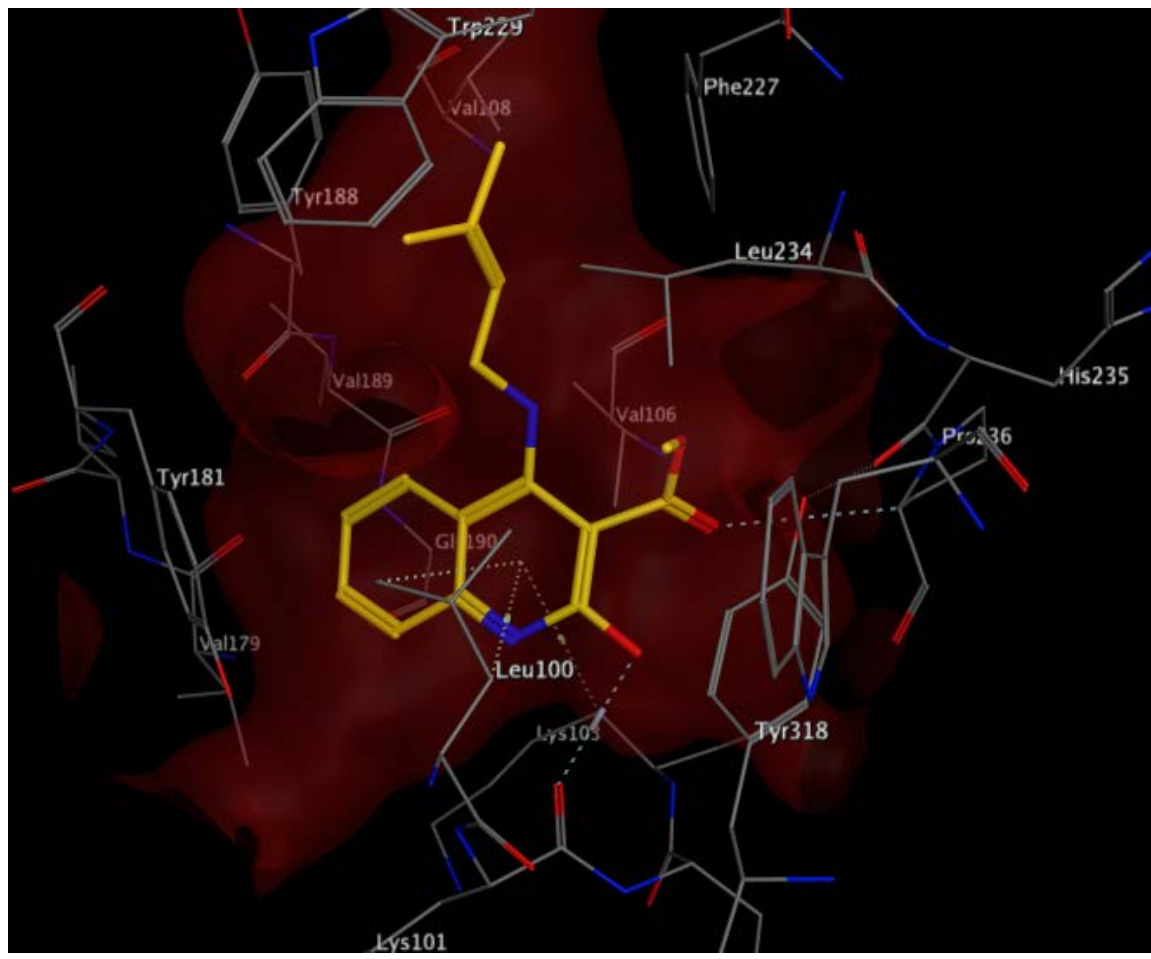

Figure 9. Docking model of compound 10 with RT PDB ID: 2B5J. It is notable the interaction between the carbonyl of ester of 10 and the backbone of Pro236.

were docked with IN. Of note, structures of compounds selected had ester and acid groups in C-3 (Figure 2) and, overall, similar functional groups as known IN inhibitors. Table 5 summarizes the docking results of the 31 best compounds. In order to select the compounds, we analyze the corresponding PLIFs fully detailed in the Supplementary material (Figure S5C). 
Table 5. Summary of docking results with IN PDB ID: 3L2U. Considering the average and the standard deviation of the docking scores, values in green font indicate the most favorable results; values in blue font indicate average scores and values in red font denote the less favored scores.

\begin{tabular}{|c|c|}
\hline ID & Docking score \\
\hline 54 & -6.48 \\
\hline 450 & -6.39 \\
\hline 86 & -6.28 \\
\hline 328 & -6.23 \\
\hline 79 & -6.21 \\
\hline 485 & -6.19 \\
\hline 52 & -6.16 \\
\hline 445 & -6.00 \\
\hline 197 & -5.90 \\
\hline 325 & -5.90 \\
\hline 16 & -5.88 \\
\hline 529 & -5.86 \\
\hline 100 & -5.68 \\
\hline 237 & -5.68 \\
\hline 211 & -5.67 \\
\hline 18 & -5.64 \\
\hline 199 & -5.63 \\
\hline 354 & -5.59 \\
\hline 249 & -5.46 \\
\hline 332 & -5.46 \\
\hline 288 & -5.41 \\
\hline 97 & -5.40 \\
\hline 244 & -5.09 \\
\hline 309 & -5.03 \\
\hline 233 & -4.83 \\
\hline 201 & -4.82 \\
\hline 206 & -4.71 \\
\hline 523 & -4.62 \\
\hline 287 & -4.58 \\
\hline 1 & -4.43 \\
\hline 10 & -4.10 \\
\hline Average & -5.53 \\
\hline Std. Dev. & \pm 0.63 \\
\hline
\end{tabular}


Similar to Table 3 and Table 4, compounds in Table 5 are sorted by increasing values of docking score (showing the best compounds at the top). The docking scores are colored by the relative magnitude with values in green indicting most favored values (e.g., better than the average plus two standard deviations) while the red values are the least favored.

Molecule 54 had the most favorable docking score. Figure 10 shows its binding model. Notably, 54 can make interactions with two $\mathrm{Mg}^{2+}$ atoms, similar to Elvitegravir. Based on this result, it is hypothesized that 54 and other molecules with favorable docking scores (Table 5) could be IN inhibitors.

\subsection{Potential Dual Inhibitors of RT and IN}

Taken together the results of the docking with RT and IN, it was concluded that 31 molecules could act as dual inhibitors. Figure 11 shows the chemical structures of the seven structures of the newly designed compounds with the best docking results (e.g., docking poses and scoring) obtained with both, RT and IN. As determined with the PDB ID: 2BAN and 2B5J RT structures, the compounds can make protein-ligand contacts with key amino acids for activity against native and mutant strains. In addition, the same seven structures are able to interact with the $\mathrm{Mg}^{2+}$ ions in the cavity between the enzyme and DNA, similar to the reports of the IN inhibitors.

\subsection{Drug-Like Properties}

The structures that were identified as potential inhibitors of RT and IN were evaluated according to the rules of Lipinski an Veber [21] [22]. Thus, for each structure were calculated the six properties of pharmaceutical interest: MW, Log $\mathrm{P}, \mathrm{HBD}$ and HBA, RB and TPSA. Results showed that all the structures comply

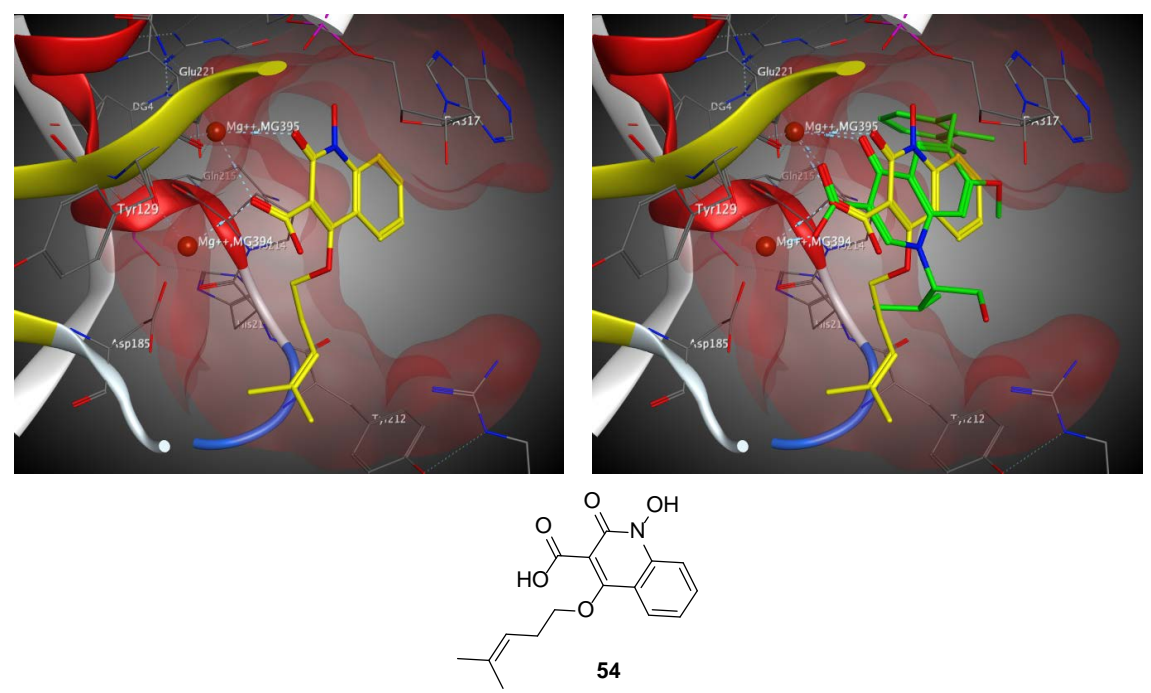

Figure 10. (Left) Docking model of 54 (carbon atoms in yellow) with IN, PBD ID: 3L2U. (Right) Proposed binding model of 54 (yellow) with Elvitegravir (carbon atoms in green). It is noteworthy the interaction with $\mathrm{Mg}^{2+}$ atoms. 
<smiles>CC(C)=CCCOc1c(C(=O)O)c(=O)n(O)c2ccccc12</smiles>

54<smiles>CCc1c(OCCC=C(C)C)c(C(=O)OC(C)C)c(=O)n(O)c1C</smiles>

450<smiles>CC(C)=CCCOc1c(C(=O)O)c(=O)[nH]c2ccc(Br)cc12</smiles>

86<smiles>CC(C)=CCCOc1c(C(=O)O)c(=O)n(O)c2ccc(I)cc12</smiles>

328<smiles>CC(C)=CCNc1c(C(=O)O)c(=O)[nH]c2ccc(I)cc12</smiles>

79<smiles>CC(C)=CCOc1c2c(n(O)c(=O)c1C(=O)O)CCC2</smiles>

485<smiles>CC(C)=CCNc1c(C(=O)O)c(=O)n(O)c2ccccc12</smiles>

Figure 11. Examples of potential dual inhibitors of RT and IN.

with the rules of Lipinski and Veber (Table S1 and Figure S4 in the Supplementary material).

\section{Conclusions and Perspectives}

Using automated molecular docking with multiple crystallographic structures of RT and IN, 31 compounds were identified as potential dual inhibitors of RT and IN. The three most promising compounds are 54, 450 and 86. All proposed compounds are synthetically accessible and have drug-like properties. The proposed compounds could have improved activity vs. currently drugs approved for the treatment of AIDS.

The major perspective of this work is the synthesis of the selected compounds and their corresponding biological evaluation as inhibitors of RT, IN and anti-HIV molecules.

\section{Acknowledgements}

A.C.V. is grateful to CONACyT and UNAM for the postdoctoral scholarship \#291053. This work was supported by the Instituto de Investigaciones Biomédicas, UNAM through the program Nuevas Alternativas de Tratamiento para Enfermedades Infecciosas (Novel Alternatives for the Treatment of Infection Diseases) (NUATEI-IIB-UNAM), and Programa de Apoyo a la Investigación y el Posgrado (PAIP) grant 5000-9163, Facultad de Química, UNAM.

\section{References}

[1] UNAIDS (2018) Fact Sheet-Latest Statistics on the Status of the AIDS Epidemic http://www.unaids.org/en/resources/fact-sheet

[2] Hopkins, A.L., Ren, J., Milton, J., et al. (2004) Design of Non-Nucleoside Inhibitors 
of HIV-1 Reverse Transcriptase with Improved Drug Resistance Properties. 1. Journal of Medicinal Chemistry, 47, 5912-5922. https://doi.org/10.1021/jm040071z

[3] Freeman, G.A., Andrews III, C.W., Hopkins, A.L., et al. (2004) Design of Non-Nucleoside Inhibitors of HIV-1 Reverse Transcriptase with Improved Drug Resistance Properties. 2. Journal of Medicinal Chemistry, 47, 5923-5936. https://doi.org/10.1021/jm040072r

[4] Medina-Franco, J.L., Martínez-Mayorga, K., Juárez-Gordiano, C. and Castillo, R. (2007) Pyridin-2(1H)-ones: A Promising Class of HIV-1 Non-Nucleoside Reverse Transcriptase Inhibitors. ChemMedChem, 2, 1141-1147. https://doi.org/10.1002/cmdc.200700054

[5] Li, A., Ouyang, Y., Wang, Z., et al. (2013) Novel Pyridinone Derivatives as Non-Nucleoside Reverse Transcriptase Inhibitors (NNRTIs) with High Potency against NNRTI-Resistant HIV-1 Strains. Journal of Medicinal Chemistry, 56, 3593-3608. https://doi.org/10.1021/jm400102x

[6] Tang, J., Maddali, K., Dreis, C.D., et al. (2011) N-3 Hydroxylation of Pyrimidine-2,4-diones Yields Dual Inhibitors of HIV Reverse Transcriptase and Integrase. ACS Medicinal Chemistry Letters, 2, 63-67. https://doi.org/10.1021/ml1002162

[7] Tang, J., Maddali, K., Pommier, Y., Sham, Y.Y. and Wang, Z. (2010) Scaffold Rearrangement of Dihydroxypyrimidine Inhibitors of HIV Integrase: Docking Model Revisited. Bioorganic and Medicinal Chemistry Letters, 20, 3275-3279.

https://doi.org/10.1016/j.bmcl.2010.04.048

[8] Murray, J.M., Kelleher, A.D. and Cooper, D.A. (2011) Timing Of the Components of the HIV Life Cycle in Productively Infected CD4+ T Cells in a Population of HIV-Infected Individuals. Journal of Virology, 85, 10798-10805.

https://doi.org/10.1128/JVI.05095-11

[9] Maartens, G., Celum, C. and Lewin, S.R. (2014) HIV Infection: Epidemiology, Pathogenesis, Treatment, and Prevention. The Lancet, 384, 258-271. https://doi.org/10.1016/S0140-6736(14)60164-1

[10] Vite-Caritino, H., Méndez-Lucio, O., Reyes, H., Cabrera, A., Chávez, D. and Medina-Franco, J.L. (2016) Advances in the Development of Pyridinone Derivatives as Non-Nucleoside Reverse Transcriptase Inhibitors. RSC Advances, 6, 2119-2130. https://doi.org/10.1039/C5RA25722K

[11] Mendez-Lucio, O., Naveja, J.J., Vite-Caritino, H., Prieto-Martinez, F.D. and Medina-Franco, J.L., Review (2013) One Drug for Multiple Targets: A Computational Perspective. Journal of the Mexican Chemical Society, 60, 168-181.

[12] U.S. Department of Health and Human Services. Antiretroviral Drugs Used in the Treatment of HIV Infection. US Food and Drug Administration. https://www.fda.gov/ForPatients/Illness/HIVAIDS/Treatment/ucm118915.htm

[13] Le Van, K., Cauvinz, C., de Walque, S., et al. (2009) New Pyridinone Derivatives as Potent HIV-1 Nonnucleoside Reverse Transcriptase Inhibitors. Journal of Medicinal Chemistry, 52, 3636-3643. https://doi.org/10.1021/jm801438e

[14] Dollé, V., Fan, E., Nguyen, C.H., et al. (1995) A New Series of Pyridinone Derivatives as Potent Non-Nucleoside Human Immunodeficiency Virus Type 1 Specific Reverse Transcriptase Inhibitors. Journal of Medicinal Chemistry, 38, 4679-4686. https://doi.org/10.1021/jm00023a007

[15] Himmel, D.M., Das, K., Clark, A.D., et al. (2005) Crystal Structures for HIV-1 Reverse Transcriptase in Complexes with Three Pyridinone Derivatives: A New Class of Non-Nucleoside Inhibitors Effective against a Broad Range of Drug-Resistant Strains. Journal of Medicinal Chemistry, 48, 7582-7591. 
https://doi.org/10.1021/jm0500323

[16] Chen, W., Zhan, P., Wu, J., Li, Z. and Liu, X. (2012) The Development of HEPT-Type HIV Non-Nucleoside Reverse Transcriptase Inhibitors and its Implications for DABO Family. Current Pharmaceutical Design, 18, 4165-4186. https://doi.org/10.2174/138161212802430440

[17] Wang, Z. and Vince, R. (2008) Design and Synthesis of Dual Inhibitors of HIV Reverse Transcriptase and Integrase: Introducing a Diketoacid Functionality into Delavirdine. Bioorganic and Medicinal Chemistry, 16, 3587-3595. https://doi.org/10.1016/j.bmc.2008.02.007

[18] Berman, H.M., Westbrook, J., Feng, Z., et al. (2000) The Protein Data Bank. Nucleic Acids Research, 28, 235-242. https://doi.org/10.1093/nar/28.1.235

[19] Hare, S., Gupta, S.S., Valkov, E., Engelman, A. and Cherepanov, P. (2010) Retroviral Intasome Assembly and Inhibition of DNA Strand Transfer. Nature, 464, 232-236. https://doi.org/10.1038/nature08784

[20] Molecular Operating Environment (MOE) (2014) Montreal, Quebec, Canada: Chemical Computing Group Inc. https://www.chemcomp.com/

[21] Krusemark, C.J. (2012) Drug Design: Structure- and Ligand-Based Approaches. The Quarterly Review of Biology, 87, 165.

[22] Kerns, E.H. and Di, L. (2016) Drug-Like Properties: Concepts, Structure Design and Methods: From ADME to Toxicity Optimization. Academic Press, Amsterdam, 31.

[23] Radi, M., Falciani, C., Contemori, L., et al. (2008) A Multidisciplinary Approach for the Identification of Novel HIV-1 Non-Nucleoside Reverse Transcriptase Inhibitors: S-DABOCs and DAVPs. ChemMedChem, 3, 573-593. https://doi.org/10.1002/cmdc.200700198

[24] Medina-Franco, J.L., Rodríguez-Morales, S., Juárez-Gordiano, C., Hernández-Campos, A., Jiménez-Barbero, J. and Castillo, R. (2004) Flexible Docking of Pyridinone Derivatives into the Non-Nucleoside Inhibitor Binding Site of HIV-1 Reverse Transcriptase. Bioorganic and Medicinal Chemistry, 12, 6085-6095. https://doi.org/10.1016/j.bmc.2004.09.008

[25] Medina-Franco, J.L., Rodríguez-Morales, S., Juárez-Gordiano, C., Hernández-Campos, A. and Castillo, R. (2004) Docking-Based CoMFA And CoMSIA Studies of Non-Nucleoside Reverse Transcriptase Inhibitors of the Pyridinone Derivative Type. Journal of Computer Aided Molecular Design, 18, 345-360. https://doi.org/10.1023/B:JCAM.0000047816.15514.ab 


\section{Supplementary Material}

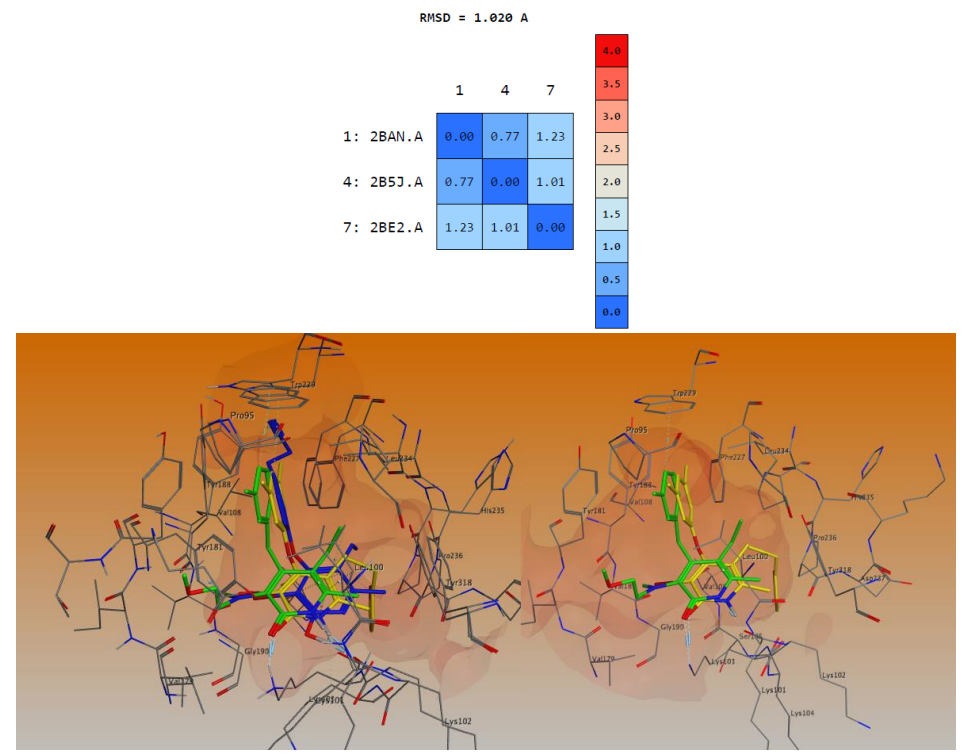

Figure S1. Superpose of RT crystallographic structures used in docking assay. Matrix of RMSD values of superposed chains. Superpose of 2BAN (green), 2B5J (blue) and 2BE2 (yellow) structures with its respective crystalline pyridinones (R157208, R165481 y R221239, respectively). Each aminoacid show the pocket. Here is possible to watch the different conformation of Tyr181 due the lateral chain of crystal of pyridinone R157208 (atoms in green color).

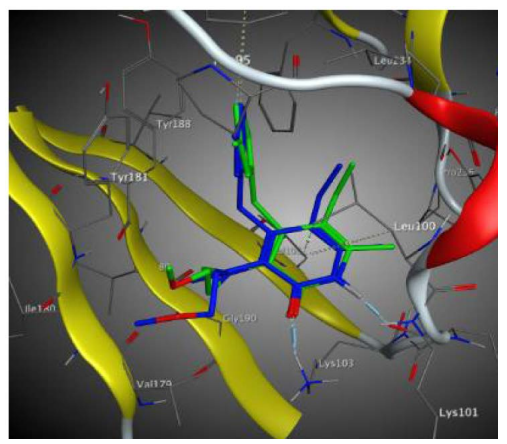

(a)

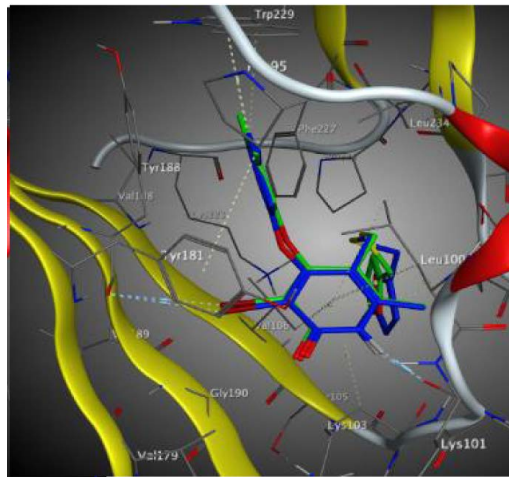

(b)

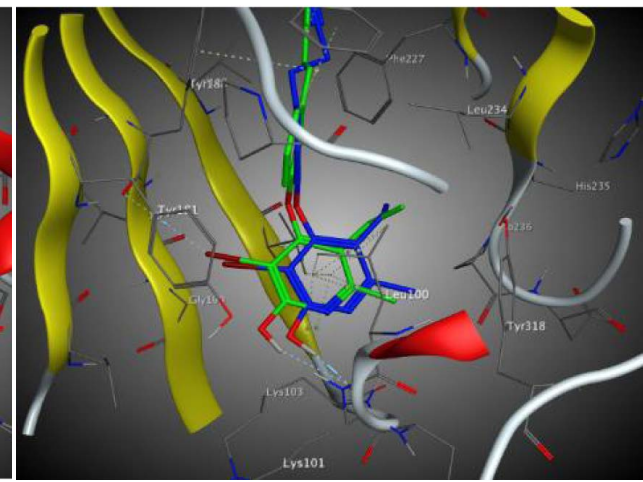

(c)

Figure S2. Re-docking of co-crystalized ligands in PDB ID: A) 2BAN, B) 2BE2 and C) 2B5J. 


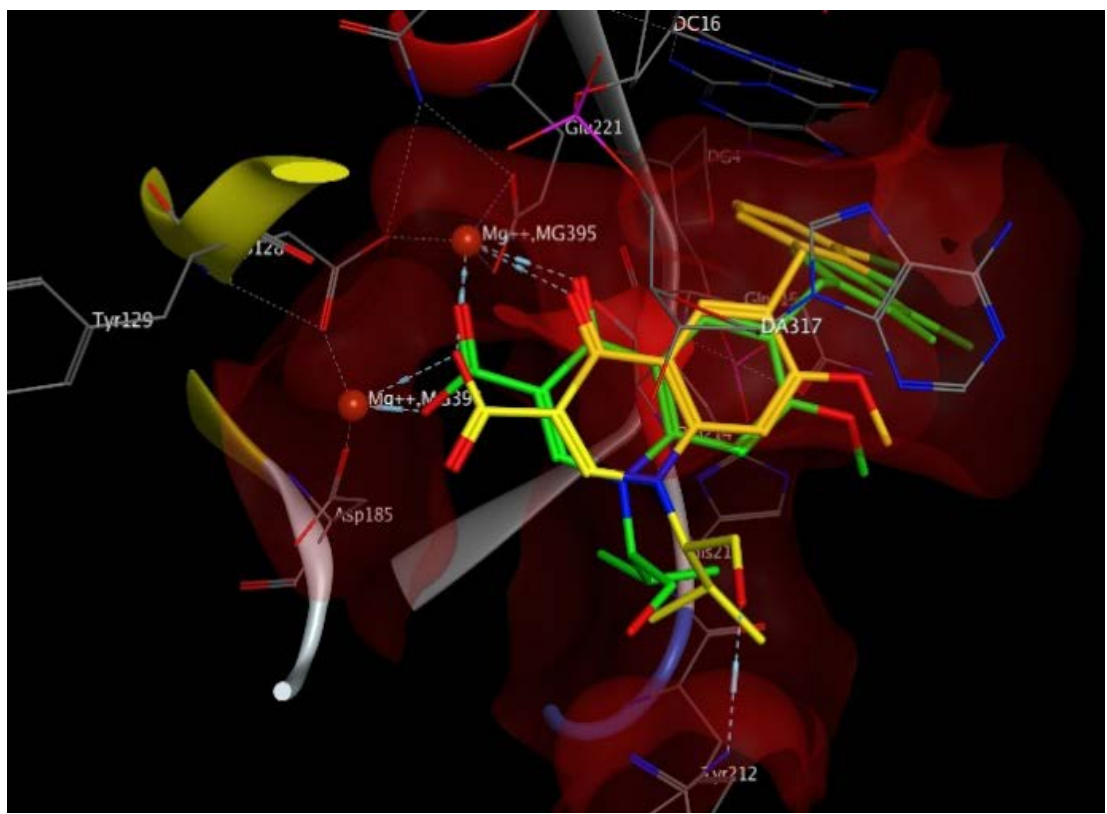

(a)

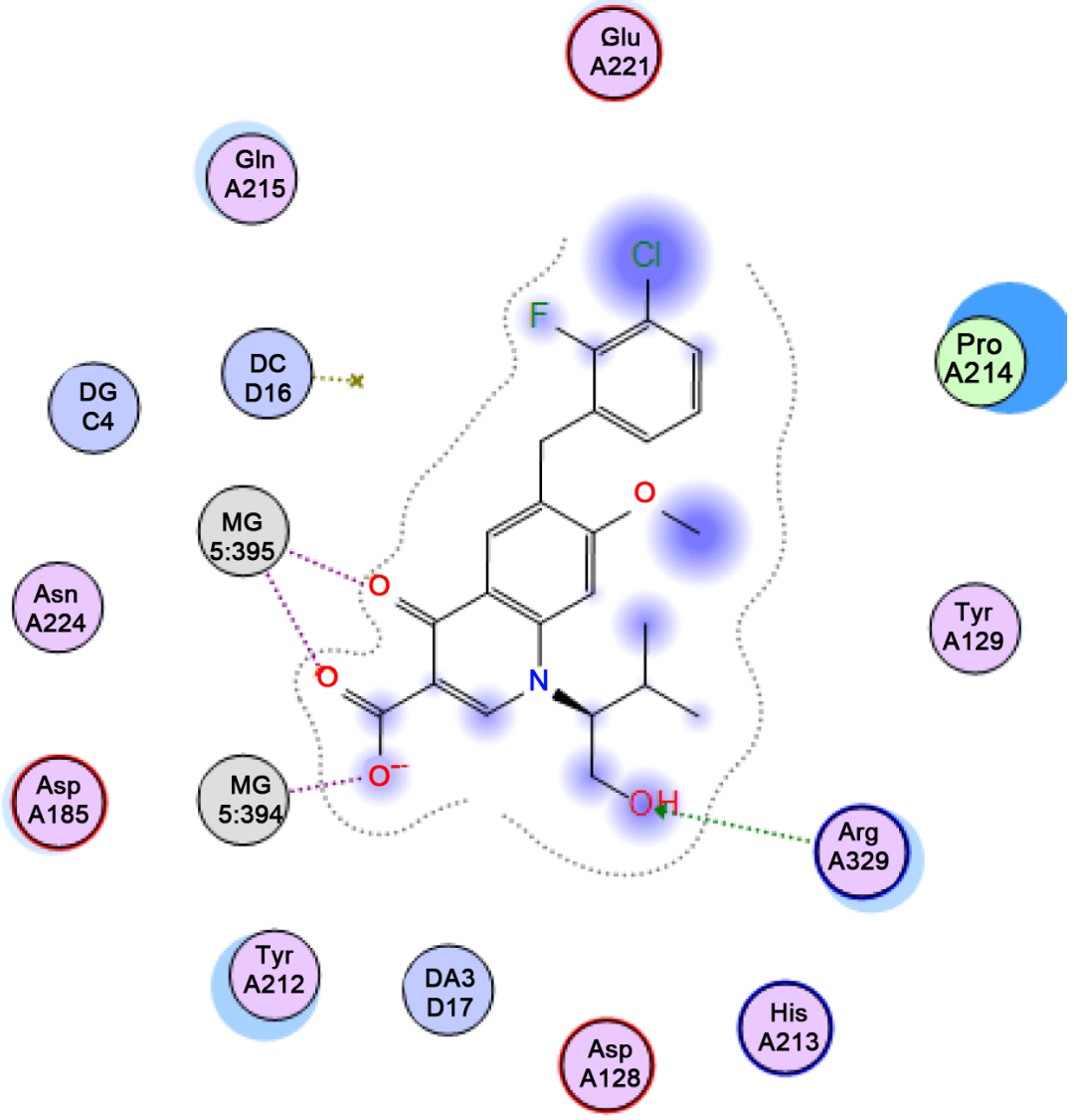

(b)

Figure S3. Re-docking of Elvitegravir with the crystallographic structure of IN, PDB ID: $3 \mathrm{~L} 2 \mathrm{U}$. The predicted binding pose is in green and the observed position in the crystallography structure is in yellow. The RMSD value was $1.297 \AA$. The figure also shows a 2D representation of the binding mode of Elvitegravir. 
Table S1. Drug-like properties of newly designed compounds as potentially inhibitors of RT an IN.

\begin{tabular}{|c|c|c|c|c|c|c|c|c|c|c|c|c|c|}
\hline Structure & MW & $\log P$ & HBD & $\mathrm{HBA}$ & $\mathrm{RB}$ & TPSA & Structure & MW & $\log P$ & HBD & HBA & $\mathrm{RB}$ & TPSA \\
\hline 1 & 314.38 & 2.6 & 2 & 2 & 6 & 67.4 & 288 & 312.21 & 2.9 & 2 & 3 & 3 & 38.3 \\
\hline 10 & 286.33 & 1.8 & 2 & 2 & 5 & 67.4 & 296 & 309.16 & 2.2 & 2 & 4 & 4 & 62.1 \\
\hline 11 & 301.34 & 2.2 & 1 & 3 & 6 & 64.6 & 299 & 257.34 & 1.2 & 3 & 3 & 4 & 64.9 \\
\hline 16 & 287.32 & 2.0 & 3 & 4 & 5 & 75.6 & 305 & 291.35 & 1.8 & 2 & 4 & 5 & 64.6 \\
\hline 18 & 270.24 & 1.2 & 3 & 5 & 5 & 99.4 & 308 & 359.21 & 3.3 & 2 & 3 & 3 & 38.3 \\
\hline 23 & 269.30 & 1.7 & 1 & 3 & 5 & 65.4 & 309 & 305.37 & 1.9 & 2 & 4 & 6 & 64.6 \\
\hline 34 & 253.30 & 1.5 & 2 & 2 & 4 & 64.9 & 310 & 290.41 & 2.1 & 2 & 3 & 5 & 41.6 \\
\hline 35 & 254.29 & 1.9 & 1 & 2 & 4 & 62.1 & 311 & 272.35 & 1.6 & 2 & 3 & 5 & 62.1 \\
\hline 38 & 251.25 & 1.2 & 1 & 3 & 5 & 85.9 & 312 & 373.23 & 3.4 & 2 & 3 & 4 & 38.3 \\
\hline 41 & 345.39 & 2.9 & 1 & 4 & 7 & 76.1 & 313 & 288.30 & 1.1 & 2 & 5 & 6 & 88.4 \\
\hline 42 & 316.31 & 1.6 & 1 & 5 & 7 & 99.9 & 315 & 255.28 & 0.8 & 2 & 4 & 5 & 85.9 \\
\hline 44 & 316.36 & 2.1 & 2 & 3 & 6 & 78.9 & 323 & 368.18 & 2.6 & 3 & 5 & 4 & 87.1 \\
\hline 49 & 317.34 & 2.1 & 1 & 4 & 6 & 76.1 & 325 & 415.18 & 3.0 & 3 & 5 & 4 & 87.1 \\
\hline 52 & 288.30 & 1.5 & 4 & 4 & 4 & 89.9 & 328 & 429.21 & 3.1 & 3 & 5 & 5 & 87.1 \\
\hline 54 & 303.31 & 1.9 & 3 & 5 & 5 & 87.1 & 332 & 409.28 & 3.4 & 2 & 3 & 6 & 78.9 \\
\hline 58 & 288.35 & 2.3 & 1 & 3 & 4 & 53.0 & 333 & 364.83 & 3.2 & 2 & 3 & 6 & 78.9 \\
\hline 59 & 302.37 & 2.4 & 1 & 3 & 5 & 53.0 & 334 & 456.28 & 3.8 & 2 & 3 & 6 & 78.9 \\
\hline 69 & 385.20 & 3.6 & 1 & 3 & 4 & 49.8 & 338 & 410.26 & 3.7 & 1 & 4 & 6 & 76.1 \\
\hline 72 & 269.30 & 1.4 & 2 & 3 & 4 & 76.4 & 340 & 457.26 & 4.1 & 1 & 4 & 6 & 76.1 \\
\hline 73 & 270.29 & 1.7 & 1 & 3 & 4 & 73.6 & 342 & 379.84 & 3.6 & 1 & 4 & 7 & 76.1 \\
\hline 74 & 284.32 & 1.8 & 1 & 3 & 5 & 73.6 & 343 & 471.29 & 4.1 & 1 & 4 & 7 & 76.1 \\
\hline 79 & 398.20 & 2.8 & 4 & 3 & 4 & 78.4 & 354 & 351.79 & 3.0 & 1 & 4 & 6 & 76.1 \\
\hline 86 & 366.21 & 2.8 & 3 & 4 & 5 & 75.6 & 356 & 410.26 & 3.3 & 1 & 4 & 7 & 76.1 \\
\hline 94 & 440.28 & 3.9 & 2 & 2 & 6 & 67.4 & 357 & 365.81 & 3.1 & 1 & 4 & 7 & 76.1 \\
\hline 95 & 379.21 & 2.5 & 1 & 4 & 7 & 88.4 & 358 & 457.26 & 3.7 & 1 & 4 & 7 & 76.1 \\
\hline 97 & 426.21 & 2.9 & 1 & 4 & 7 & 88.4 & 362 & 381.23 & 2.6 & 2 & 3 & 5 & 78.9 \\
\hline 99 & 349.81 & 3.6 & 1 & 3 & 6 & 64.6 & 364 & 428.23 & 2.9 & 2 & 3 & 5 & 78.9 \\
\hline 100 & 441.26 & 4.2 & 1 & 3 & 6 & 64.6 & 372 & 351.79 & 2.7 & 1 & 4 & 6 & 76.1 \\
\hline 101 & 408.29 & 3.9 & 1 & 3 & 7 & 64.6 & 382 & 399.19 & 2.3 & 1 & 4 & 5 & 76.8 \\
\hline 102 & 363.84 & 3.7 & 1 & 3 & 7 & 64.6 & 383 & 367.24 & 3.1 & 1 & 3 & 4 & 53.0 \\
\hline 113 & 380.24 & 3.3 & 1 & 3 & 6 & 64.6 & 392 & 348.20 & 2.3 & 2 & 3 & 4 & 76.4 \\
\hline 114 & 335.79 & 3.1 & 1 & 3 & 6 & 64.6 & 394 & 395.20 & 2.7 & 2 & 3 & 4 & 76.4 \\
\hline 120 & 332.74 & 2.4 & 1 & 4 & 7 & 88.4 & 402 & 318.76 & 2.5 & 1 & 3 & 5 & 73.6 \\
\hline 124 & 412.23 & 3.1 & 2 & 2 & 5 & 67.4 & 423 & 404.63 & 3.9 & 2 & 2 & 3 & 52.6 \\
\hline 132 & 335.79 & 2.9 & 1 & 3 & 6 & 64.6 & 429 & 405.62 & 4.2 & 1 & 3 & 3 & 49.8 \\
\hline 133 & 427.24 & 3.4 & 1 & 3 & 6 & 64.6 & 433 & 511.10 & 4.9 & 1 & 3 & 4 & 49.8 \\
\hline
\end{tabular}




\section{Continued}

\begin{tabular}{|c|c|c|c|c|c|c|c|c|c|c|c|c|c|}
\hline 146 & 365.27 & 3.3 & 1 & 2 & 5 & 41.6 & 439 & 308.38 & 1.7 & 2 & 3 & 7 & 78.9 \\
\hline 151 & 395.20 & 2.9 & 1 & 3 & 5 & 65.4 & 442 & 308.33 & 1.2 & 1 & 5 & 8 & 99.9 \\
\hline 153 & 287.75 & 2.2 & 2 & 2 & 4 & 64.9 & 445 & 281.31 & 1.4 & 3 & 5 & 5 & 87.1 \\
\hline 156 & 273.68 & 1.2 & 1 & 3 & 5 & 85.9 & 446 & 323.39 & 2.5 & 1 & 4 & 7 & 76.1 \\
\hline 161 & 347.21 & 2.8 & 1 & 2 & 5 & 62.1 & 447 & 309.36 & 2.0 & 1 & 4 & 7 & 76.1 \\
\hline 162 & 302.76 & 2.6 & 1 & 2 & 5 & 62.1 & 448 & 316.20 & 2.8 & 1 & 3 & 4 & 49.8 \\
\hline 169 & 433.09 & 4.2 & 2 & 1 & 3 & 41.1 & 450 & 337.42 & 2.6 & 1 & 4 & 8 & 76.1 \\
\hline 173 & 387.07 & 4.1 & 1 & 2 & 3 & 38.3 & 451 & 323.39 & 2.1 & 1 & 4 & 8 & 76.1 \\
\hline 177 & 356.65 & 4.0 & 1 & 2 & 4 & 38.3 & 454 & 320.35 & 1.8 & 1 & 5 & 8 & 99.9 \\
\hline 178 & 448.10 & 4.6 & 1 & 2 & 4 & 38.3 & 459 & 261.32 & 1.1 & 2 & 3 & 5 & 76.4 \\
\hline 189 & 389.62 & 4.3 & 1 & 2 & 3 & 38.3 & 463 & 247.25 & 0.2 & 1 & 4 & 6 & 97.3 \\
\hline 190 & 481.07 & 4.9 & 1 & 2 & 3 & 38.3 & 470 & 294.39 & 2.0 & 1 & 3 & 6 & 53.0 \\
\hline 192 & 389.62 & 4.3 & 1 & 2 & 3 & 38.3 & 472 & 377.22 & 3.3 & 1 & 3 & 5 & 49.8 \\
\hline 193 & 481.07 & 4.9 & 1 & 2 & 3 & 38.3 & 473 & 292.29 & 1.0 & 1 & 5 & 7 & 99.9 \\
\hline 194 & 431.03 & 3.8 & 1 & 3 & 4 & 62.1 & 474 & 277.32 & 1.2 & 1 & 4 & 6 & 76.8 \\
\hline 197 & 264.32 & 1.2 & 5 & 4 & 5 & 78.4 & 475 & 259.26 & 0.7 & 1 & 4 & 6 & 97.3 \\
\hline 199 & 292.38 & 1.8 & 3 & 3 & 7 & 67.4 & 485 & 279.29 & 1.0 & 3 & 5 & 4 & 87.1 \\
\hline 201 & 250.25 & 0.3 & 4 & 6 & 6 & 99.4 & 486 & 321.37 & 2.0 & 1 & 4 & 6 & 76.1 \\
\hline 206 & 307.39 & 2.6 & 2 & 4 & 7 & 64.6 & 487 & 307.35 & 1.6 & 1 & 4 & 6 & 76.1 \\
\hline 210 & 321.42 & 2.7 & 2 & 4 & 8 & 64.6 & 496 & 311.13 & 1.7 & 1 & 4 & 4 & 73.6 \\
\hline 211 & 307.39 & 2.2 & 2 & 4 & 8 & 64.6 & 499 & 259.31 & 0.6 & 2 & 3 & 4 & 76.4 \\
\hline 212 & 314.22 & 3.0 & 2 & 3 & 5 & 38.3 & 503 & 245.24 & -0.3 & 1 & 4 & 5 & 97.3 \\
\hline 219 & 245.33 & 1.2 & 3 & 3 & 5 & 64.9 & 505 & 293.32 & 1.2 & 1 & 4 & 5 & 76.1 \\
\hline 224 & 332.14 & 2.1 & 2 & 4 & 5 & 62.1 & 506 & 278.35 & 1.5 & 1 & 3 & 4 & 53.0 \\
\hline 225 & 279.34 & 1.8 & 2 & 4 & 6 & 64.6 & 507 & 260.29 & 0.9 & 1 & 3 & 4 & 73.6 \\
\hline 226 & 264.37 & 2.0 & 2 & 3 & 5 & 41.6 & 513 & 290.27 & 0.5 & 1 & 5 & 6 & 99.9 \\
\hline 227 & 246.31 & 1.5 & 2 & 3 & 5 & 62.1 & 515 & 257.25 & 0.3 & 1 & 4 & 5 & 97.3 \\
\hline 228 & 347.20 & 3.3 & 2 & 3 & 4 & 38.3 & 523 & 306.32 & 0.8 & 1 & 5 & 7 & 99.9 \\
\hline 231 & 260.34 & 1.6 & 2 & 3 & 6 & 62.1 & 525 & 293.32 & 1.4 & 3 & 5 & 4 & 87.1 \\
\hline 233 & 276.29 & 1.1 & 2 & 5 & 7 & 88.4 & 526 & 335.40 & 2.5 & 1 & 4 & 6 & 76.1 \\
\hline 235 & 243.27 & 0.8 & 2 & 4 & 6 & 85.9 & 529 & 307.35 & 1.5 & 3 & 5 & 5 & 87.1 \\
\hline 236 & 344.15 & 2.6 & 2 & 4 & 5 & 62.1 & 531 & 335.40 & 2.1 & 1 & 4 & 7 & 76.1 \\
\hline 237 & 262.31 & 0.7 & 5 & 4 & 4 & 78.4 & 535 & 318.33 & 1.3 & 1 & 5 & 7 & 99.9 \\
\hline 244 & 283.13 & 1.2 & 2 & 4 & 4 & 62.1 & 538 & 291.39 & 1.6 & 2 & 2 & 4 & 55.8 \\
\hline 246 & 305.37 & 2.1 & 2 & 4 & 6 & 64.6 & 539 & 273.34 & 1.1 & 2 & 3 & 4 & 76.4 \\
\hline 249 & 277.32 & 1.1 & 4 & 5 & 5 & 75.6 & 540 & 374.22 & 2.9 & 2 & 2 & 3 & 52.6 \\
\hline
\end{tabular}




\section{Continued}

\begin{tabular}{cccccccccccccc}
\hline 250 & 319.40 & 2.2 & 2 & 4 & 7 & 64.6 & 541 & 292.29 & 0.4 & 1 & 5 & 6 & 99.9 \\
252 & 312.21 & 2.5 & 2 & 3 & 4 & 38.3 & 542 & 277.32 & 0.7 & 1 & 4 & 5 & 76.8 \\
259 & 243.31 & 0.7 & 3 & 3 & 4 & 64.9 & 546 & 292.38 & 1.9 & 1 & 3 & 4 & 53.0 \\
267 & 244.29 & 1.0 & 2 & 3 & 4 & 62.1 & 548 & 375.21 & 3.2 & 1 & 3 & 3 & 49.8 \\
268 & 345.18 & 2.8 & 2 & 3 & 3 & 38.3 & 550 & 306.41 & 2.0 & 1 & 3 & 5 & 53.0 \\
269 & 291.35 & 1.4 & 2 & 4 & 6 & 64.6 & 551 & 288.35 & 1.5 & 1 & 3 & 5 & 73.6 \\
271 & 258.32 & 1.1 & 2 & 3 & 5 & 62.1 & 554 & 289.33 & 1.2 & 1 & 4 & 5 & 76.8 \\
287 & 305.37 & 2.1 & 2 & 4 & 6 & 64.6 & 555 & 271.28 & 0.7 & 1 & 4 & 5 & 97.3 \\
Specification & $<500$ & $<5$ & $<5$ & $<10$ & $<10$ & $<140$ & Specification & $<500$ & $<5$ & $<5$ & $<10$ & $<10$ & $<140$ \\
\hline
\end{tabular}
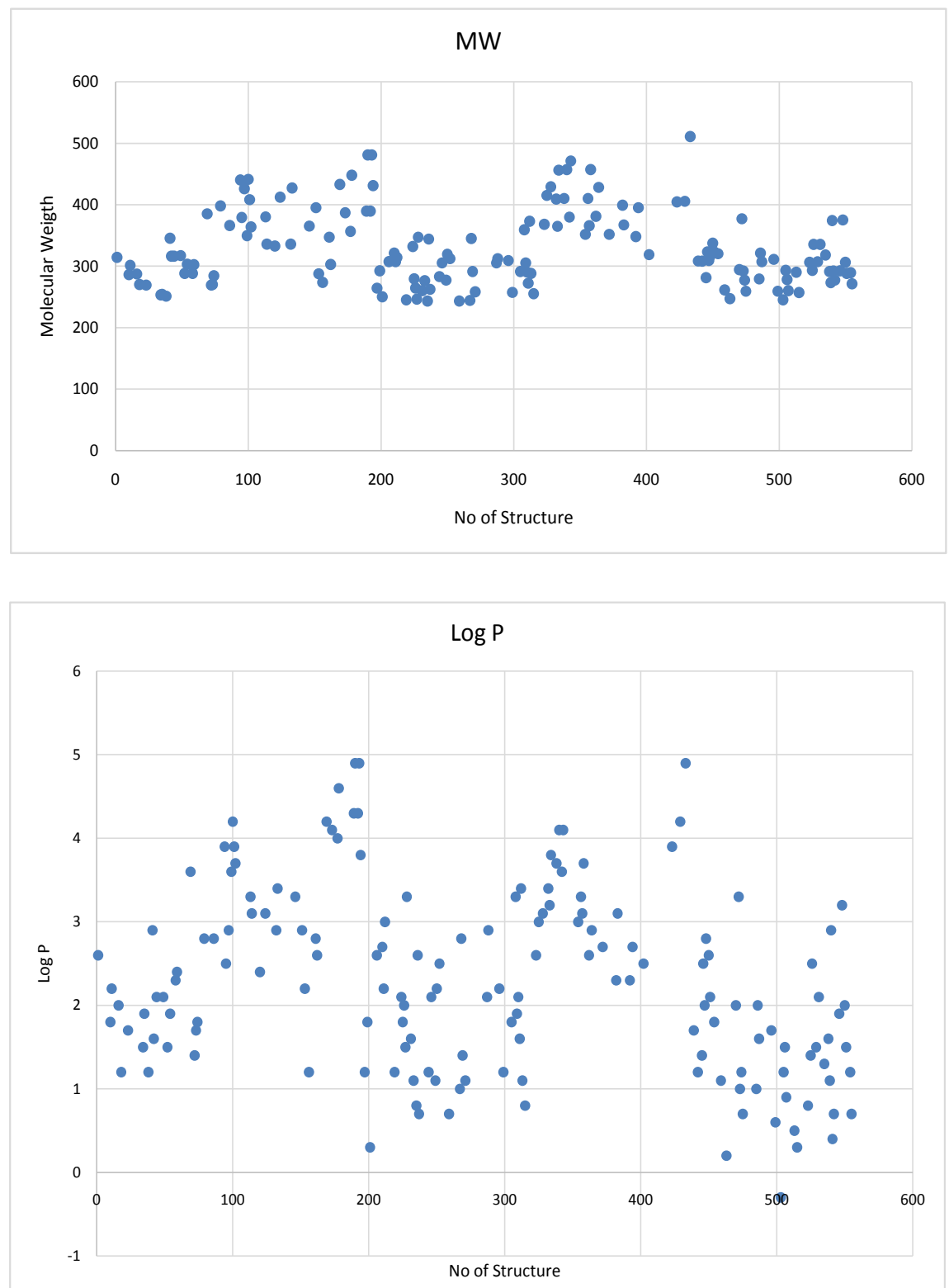

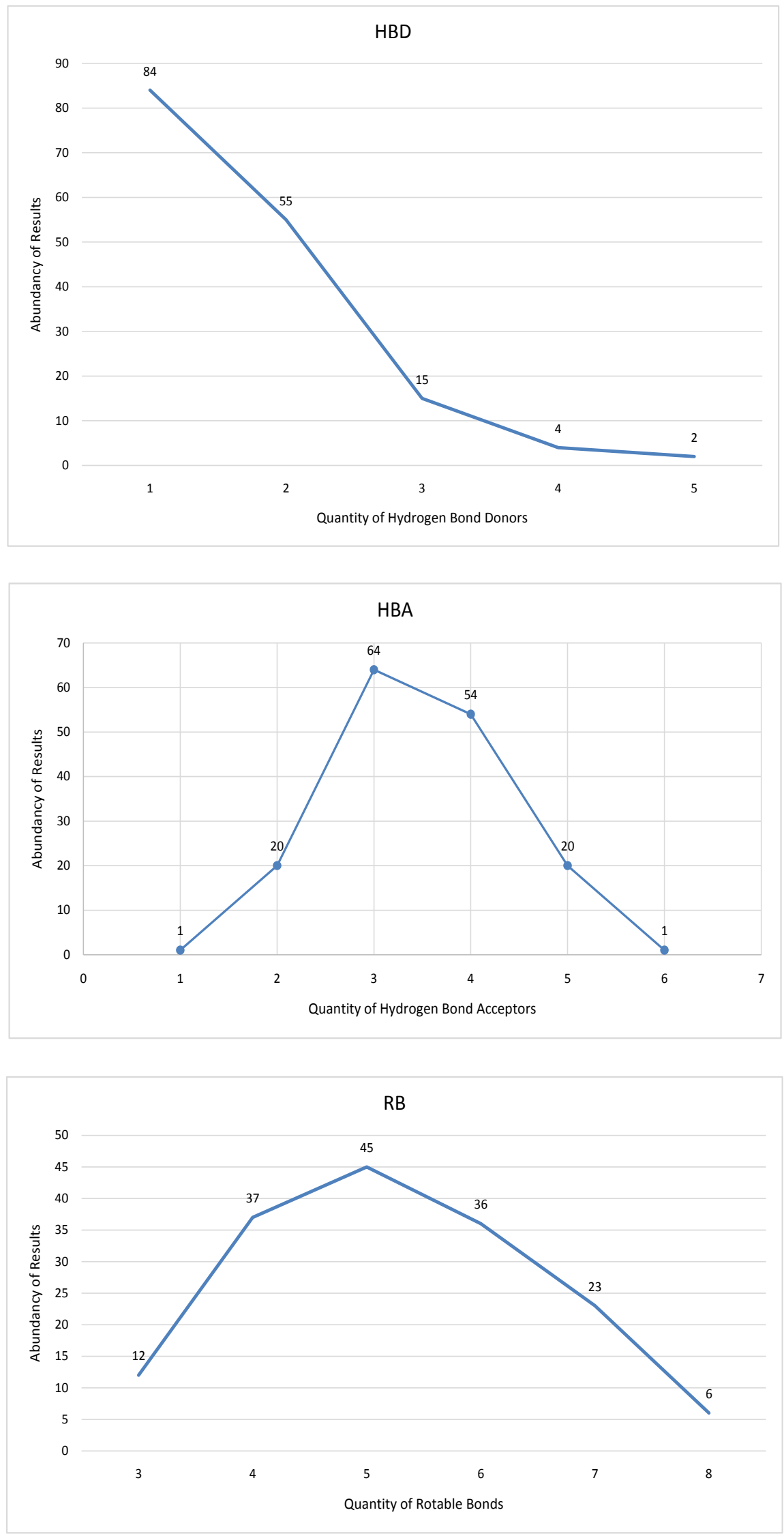


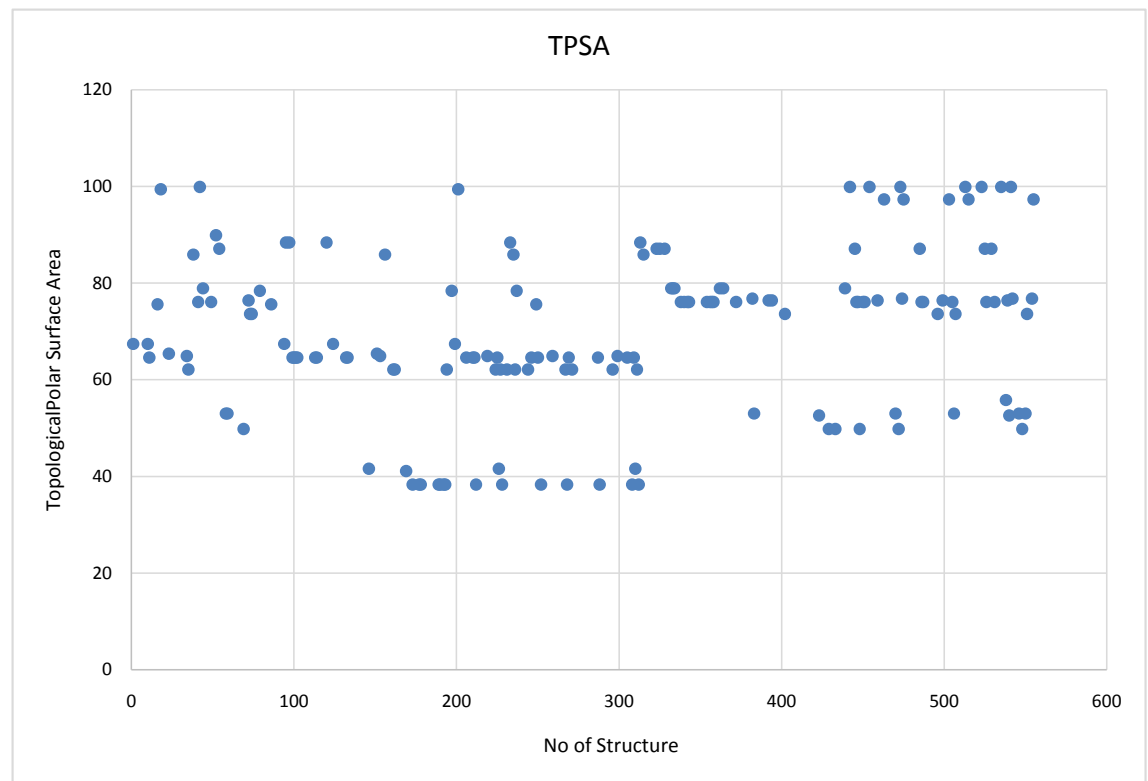

Figure S4. Distribution of the drug-like properties of newly designed compounds (see also TableS1).

\section{Analysis of PLIFS}

\section{PLIF of docking results with RT (PDB: $2 B A N$ and PDB: $2 B 5$ )}

The Protein Ligand Interaction Fingerprint (PLIF) generated with MOE was important to select the structures with interactions of interest when the docking has finished. According with the information obtained from previous studies a mayor feature of the pyridinone and related compound as inhibitors of RT of HIV is the interaction between carbonyl of Lys101 in RT and the hydrogen of amine in pyridinone through hydrogen bond. This feature is the key to be fixed the pyridinone in the RT pocket. In the analysis of PLIFs from the docking models, we were looking for this interaction key and at the same time the interaction of substituents in C-3 and C-4 with conserved amino acids in RT. In particular, is known that compounds maintaining interaction with Trp229, Tyr318 and Pro236 in RT could be good inhibitors of HIV. To docking studies were taken in count two crystallographic structures of RT and each PDB crystalline structure (2BAN and 2B5J) have a co-crystalized pyridinone, but the last one (PDB ID: 2B5J) has a tautomeric pyridinone, so in this conformation were tested the structures.

Once obtained the data base of results of docking with RT the next step was to generate in MOE the PLIF for PDB: 2BAN. When was selected the Lys101 backbone donor interaction in PLIF, MOE generated a list of conformations with the hydrogen bond between hydrogen of the amine of pyridinone and oxygen of carbonyl group of Lys101 (Figure S5A). The sum of conformations that implied Lys101 was 2790 and come from a total of 14,828 conformations. The next step was to verify which structures with the best results interact with the amino acids Tyr181, Tyr188, Trp229, Pro236 and Tyr318. To identify the struc- 
tures that interact with the amino acids before mentioned was necessary analyze visually the 2790 structures and 83 structures were selected for docking with 2BAN.

For the docking results with PDB ID: 2B5J it was generated a list using data of PLIF where was selected the Lys101 backbone donor interaction (Figure S5B). The list of conformations that implied Lys101 had 2,157 conformations from a total of 13,548 conformations. As next step 2157 posed were analyzed obtaining 91 structures with interaction with Tyr181, Tyr188, Trp229, Pro236 and Tyr318.

PLIF of docking results with IN (PDB: $3 L 2 U$ )

Once we selected 76 structures with similar structural characteristics to Elvitegravir and performed the docking, PLIFs were generated with MOE. The goal was to identify those compounds with similar interactions as the reference co-crystal ligand, Elvitegravir, in particular the interactions with $\mathrm{Mg}^{2+}$ ions. The docking simulations gave 1862 conformations and was generated the PLIF in MOE. The data of PLIF were organized to get a list of conformations that interact with two $\mathrm{Mg}^{2+}$ and where listed 187 conformations of compounds that had interaction at the same time with two atoms of $\mathrm{Mg}^{2+}$ (Figure S5C).

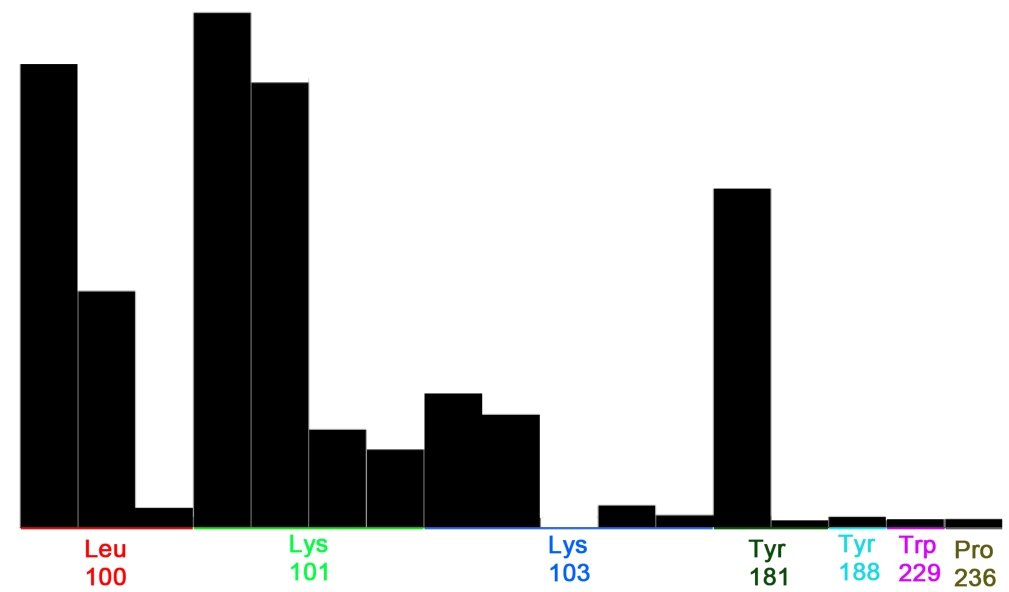

(a)

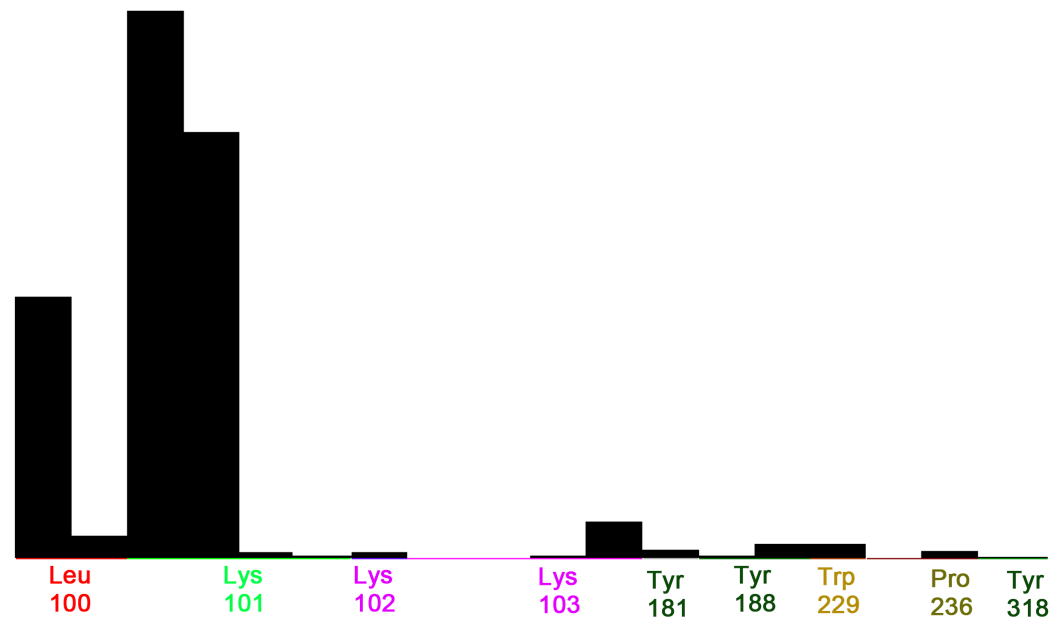

(b) 


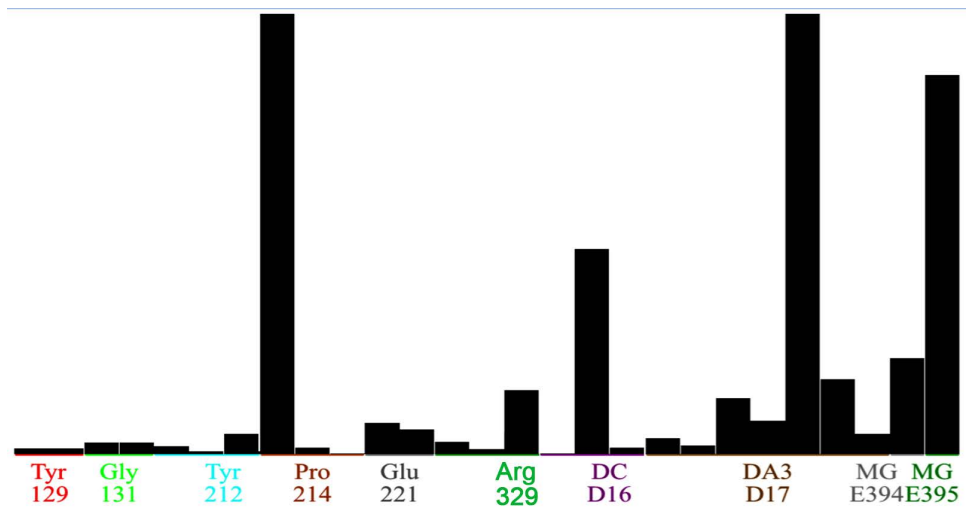

(c)

Figure S5. PLIF of results of docking of pyridinone structures and (a) 2BAN, (b) 2BJ5, and (c) $3 \mathrm{~L} 2 \mathrm{U}$.

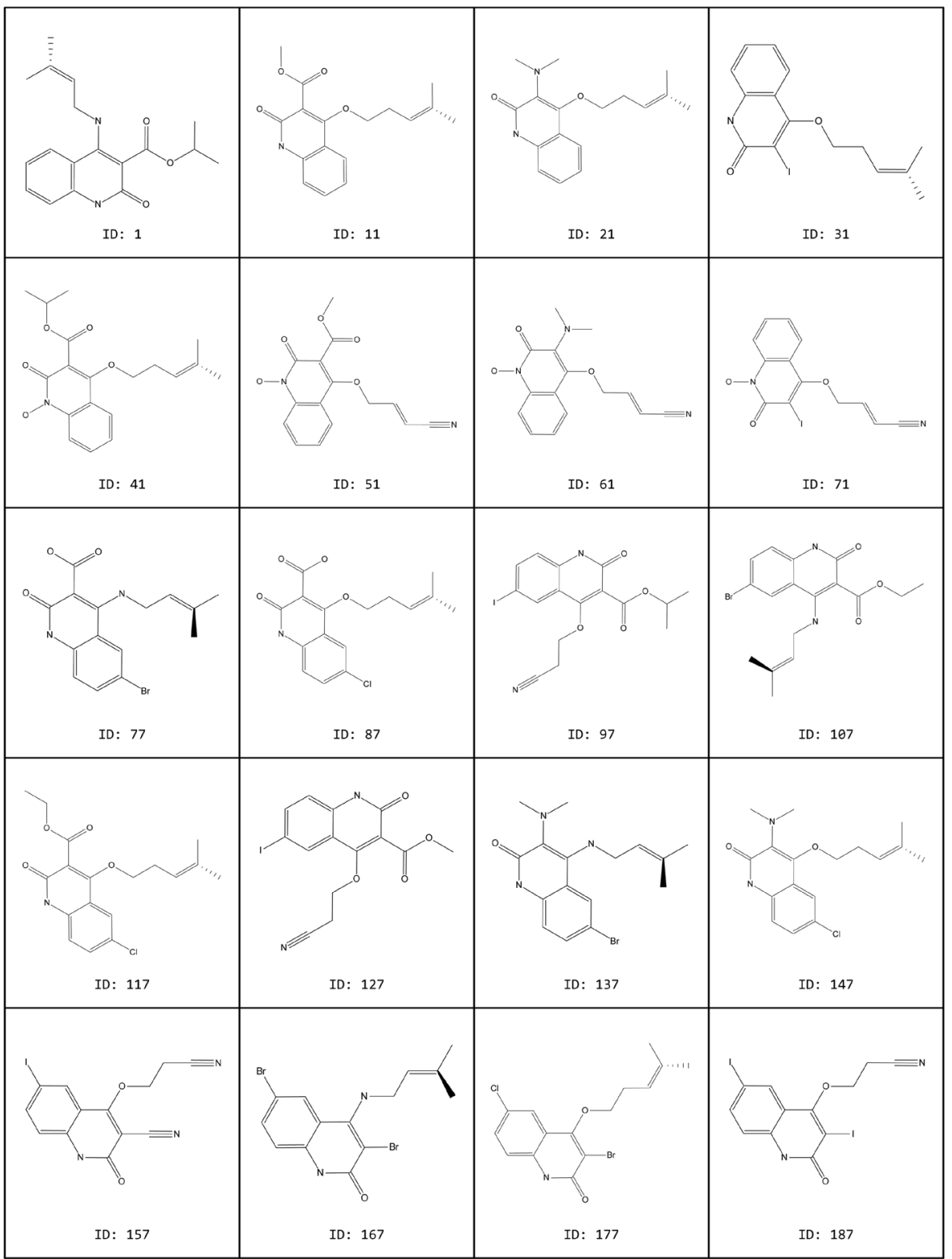




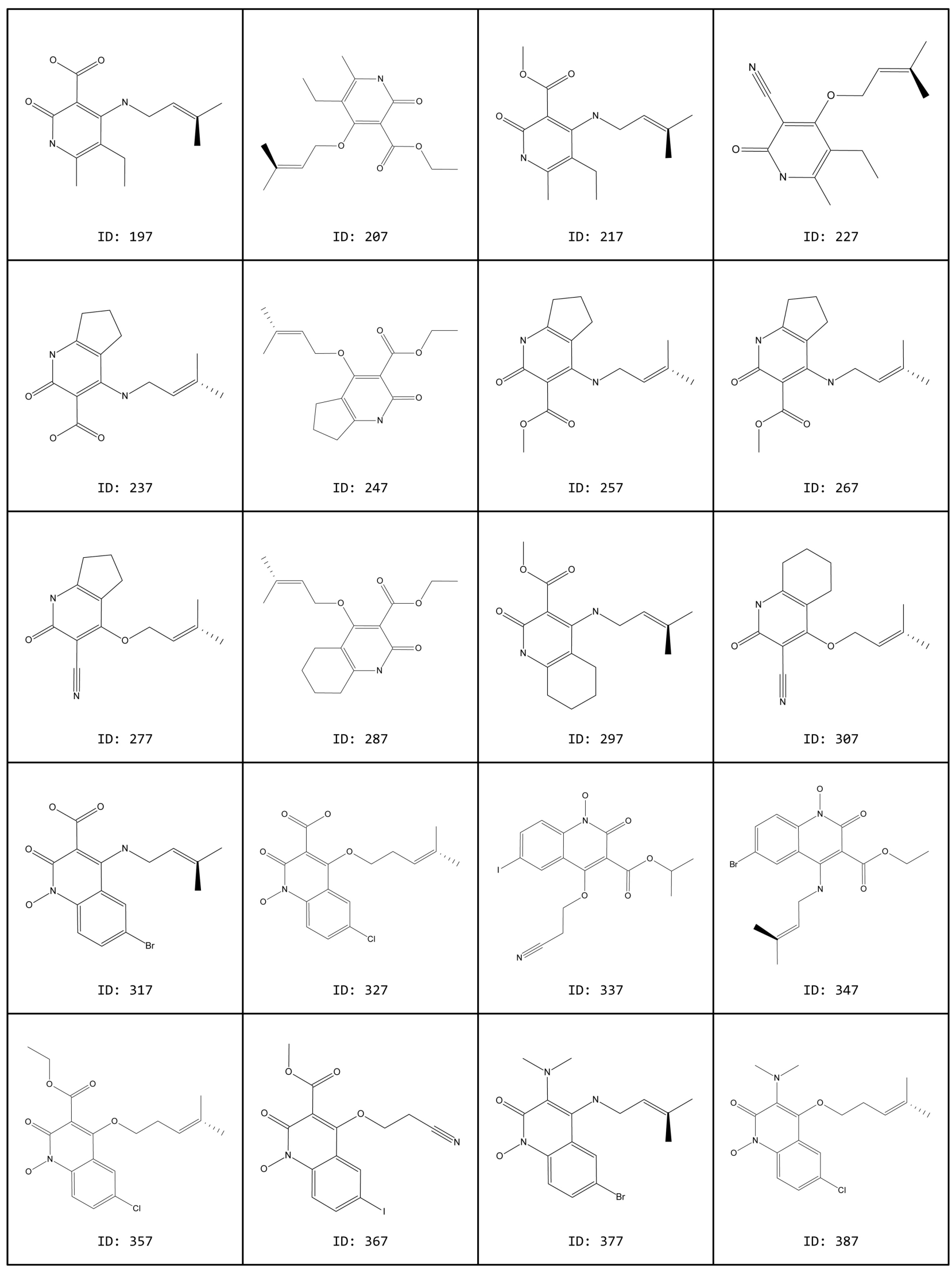




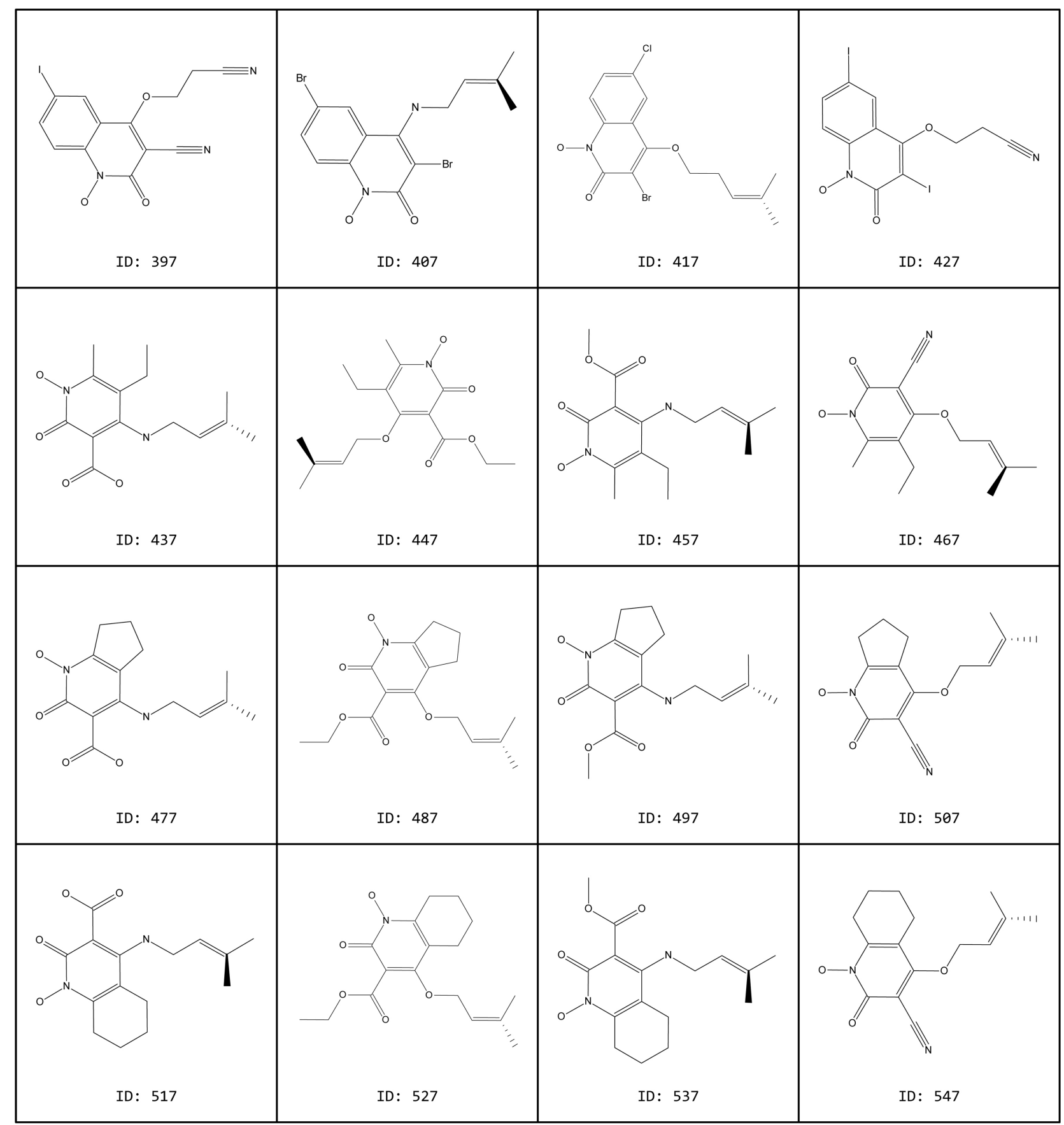

Figure S6. 56 structures aligned with co-crystalized pyridinones. 


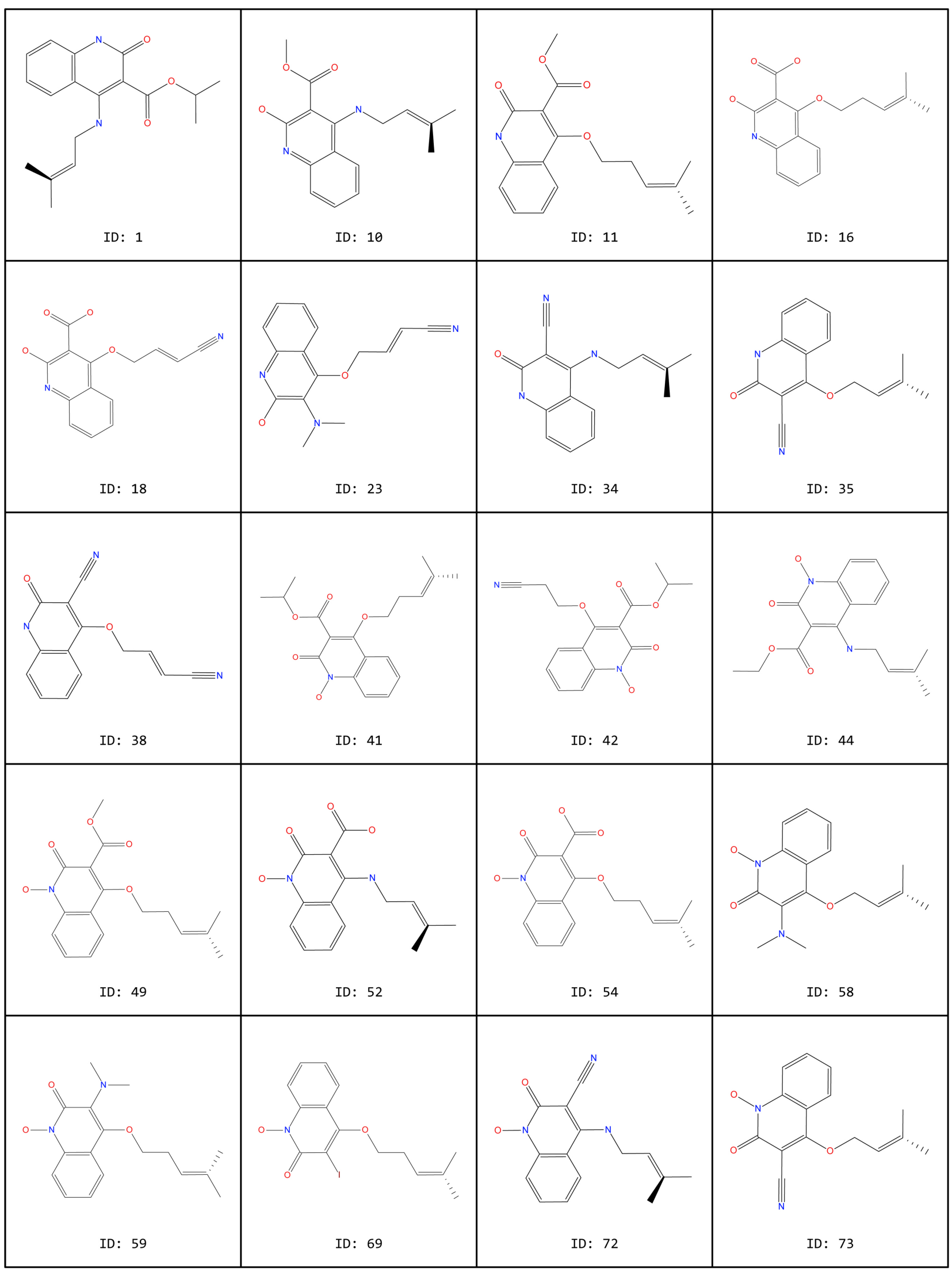




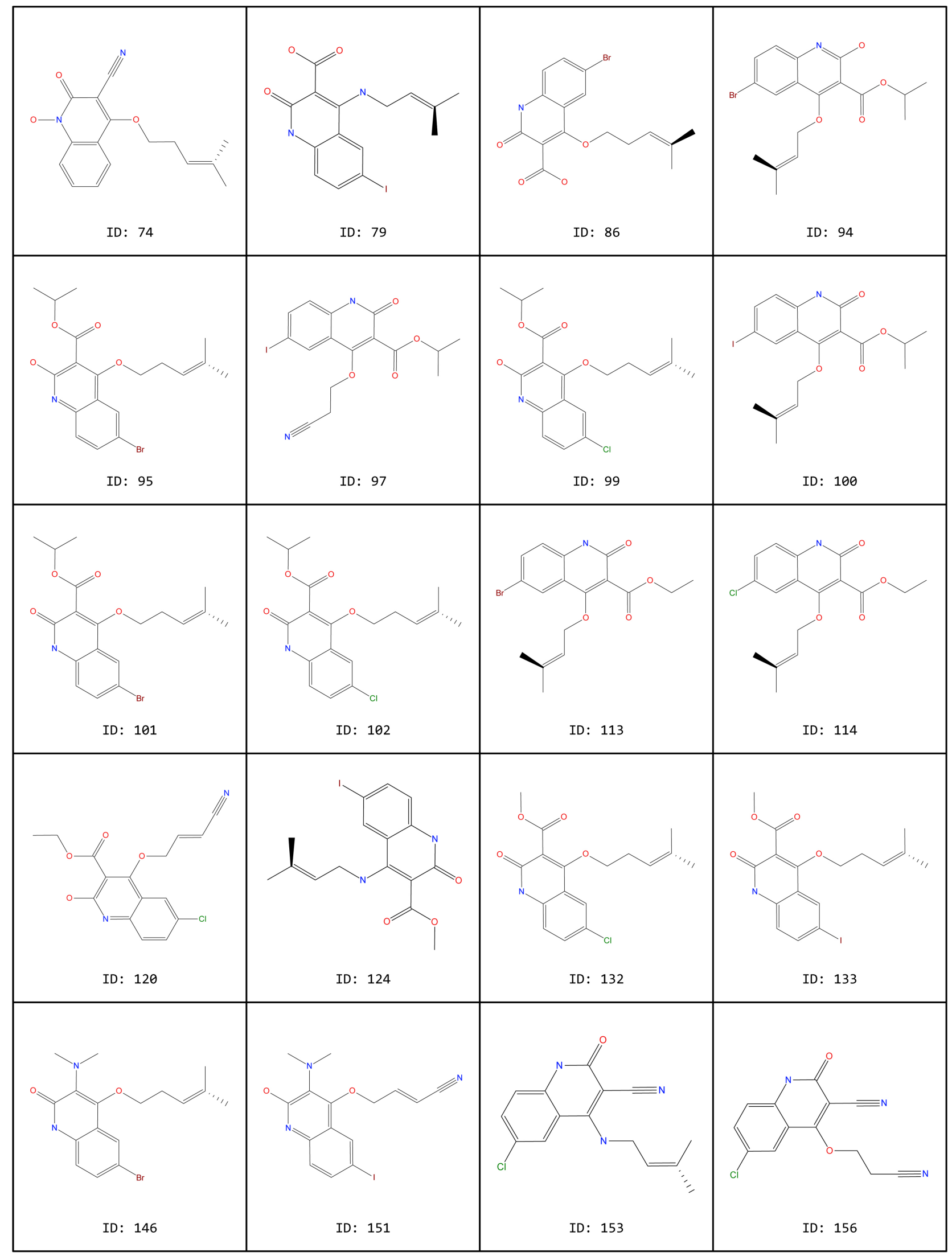




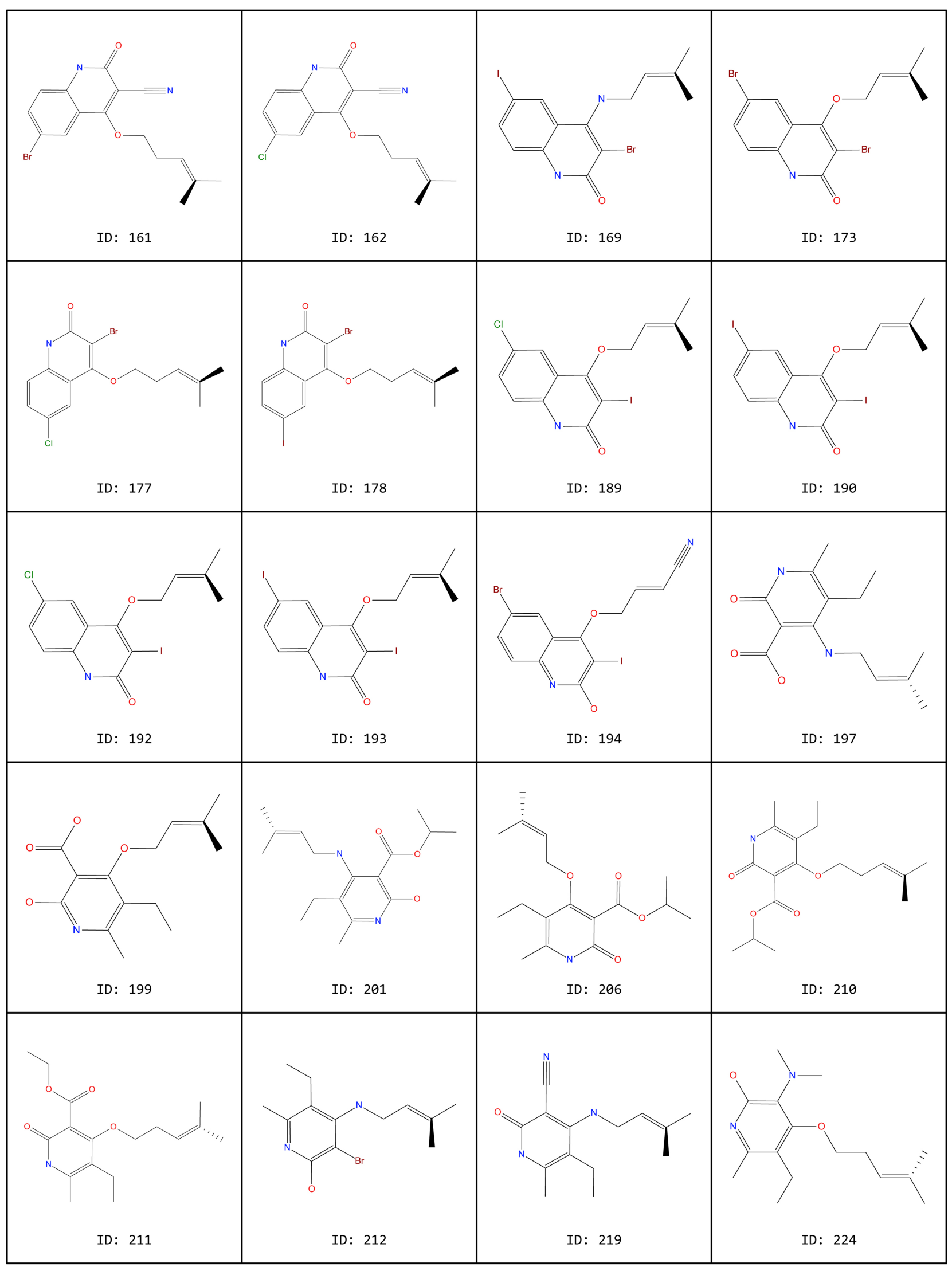




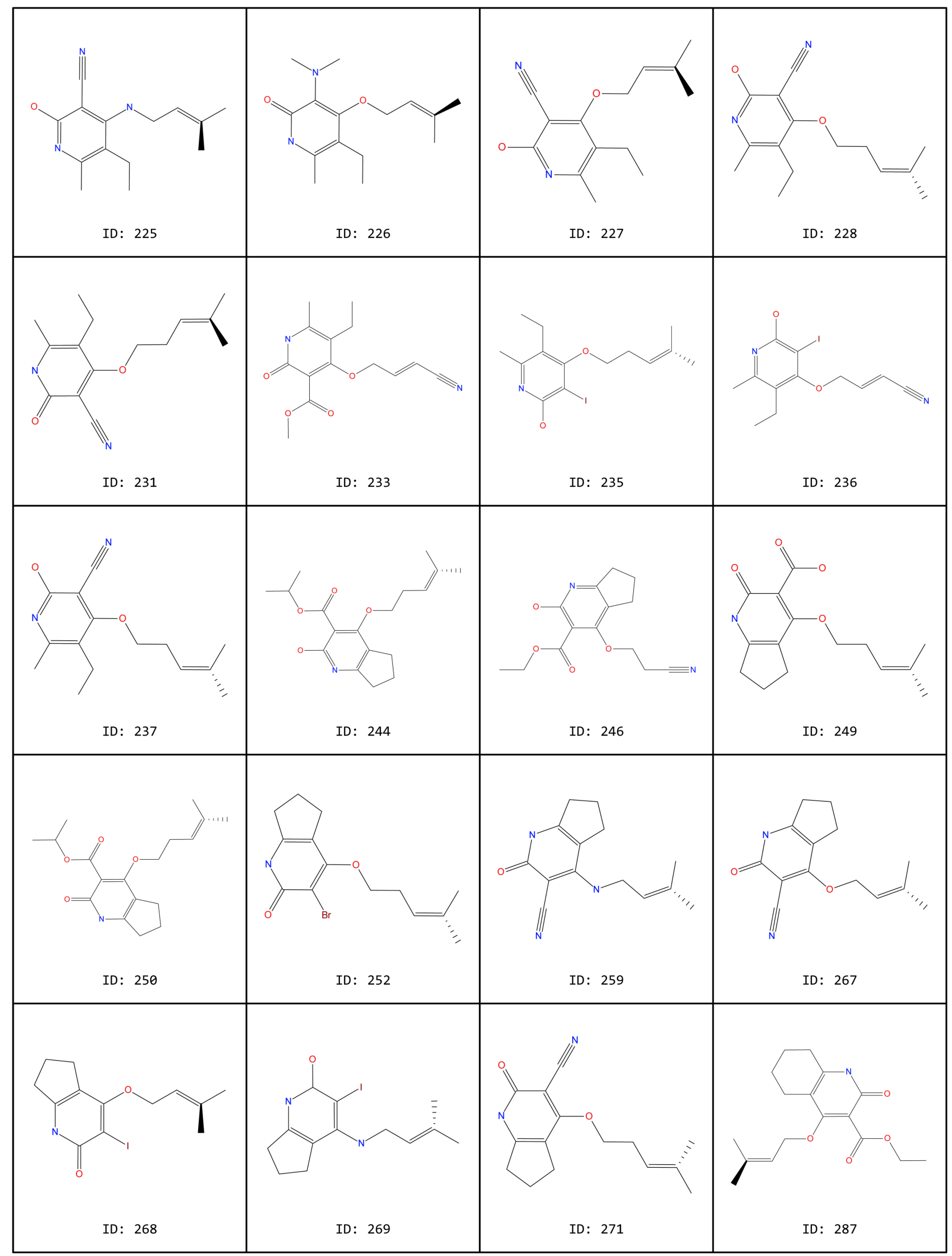




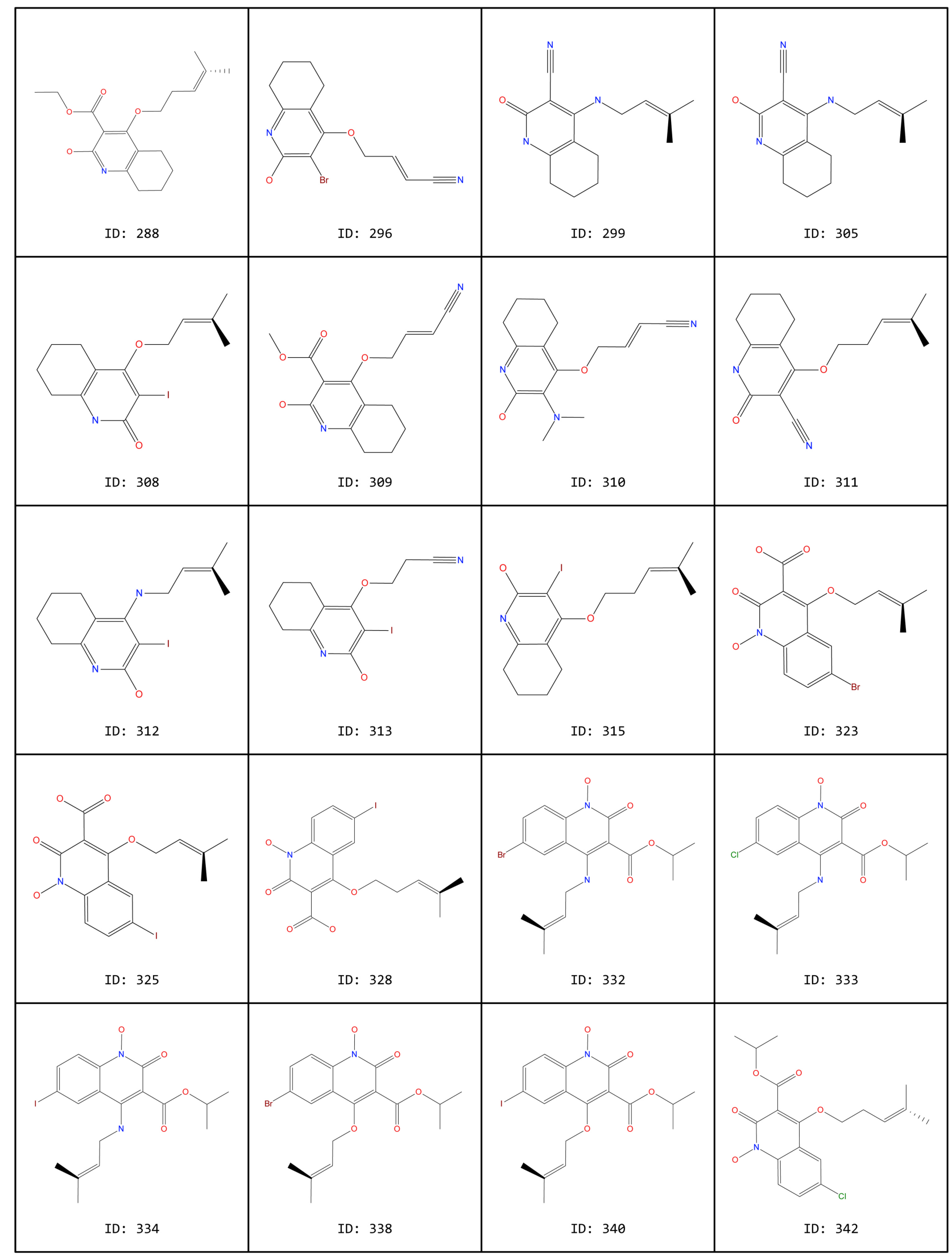




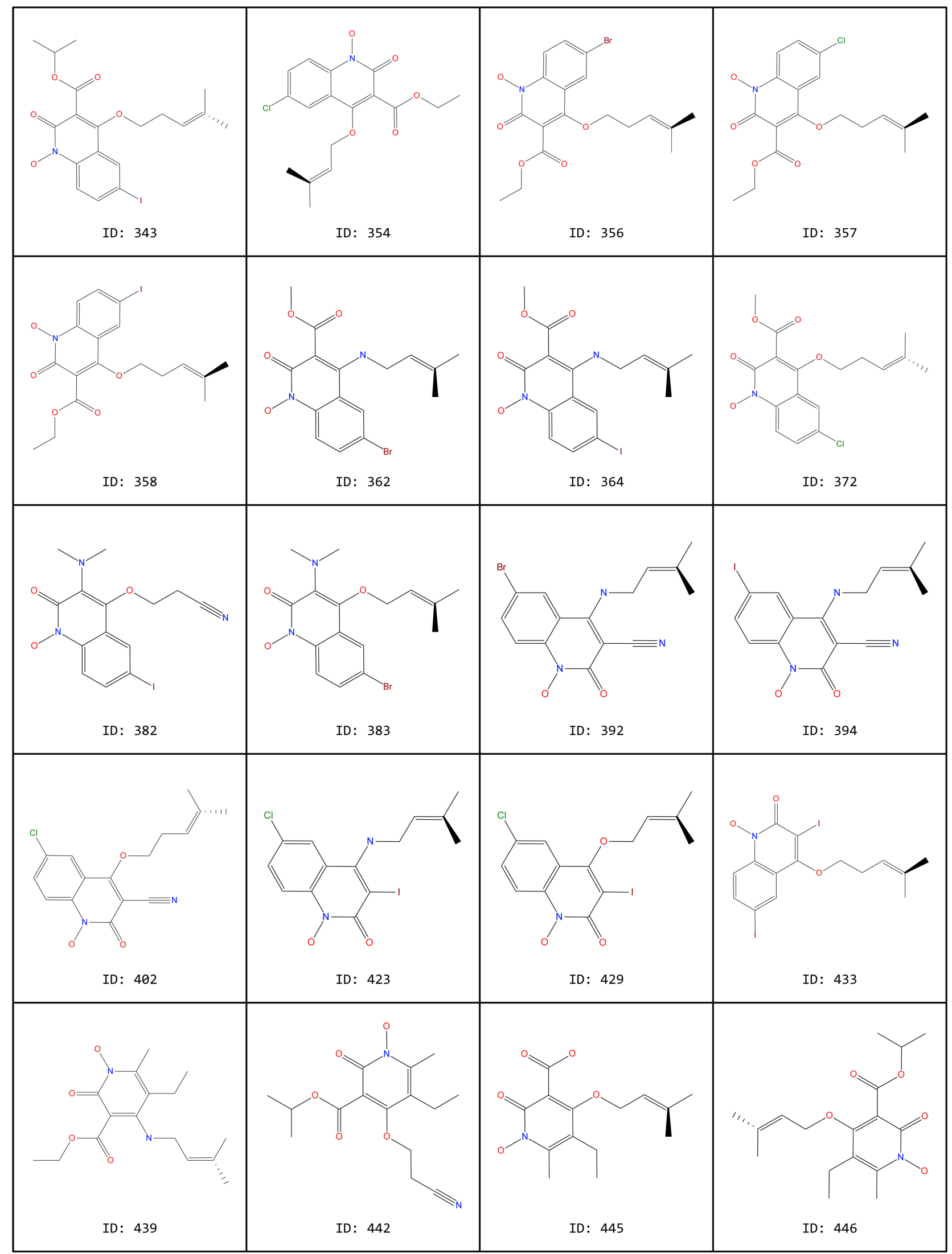




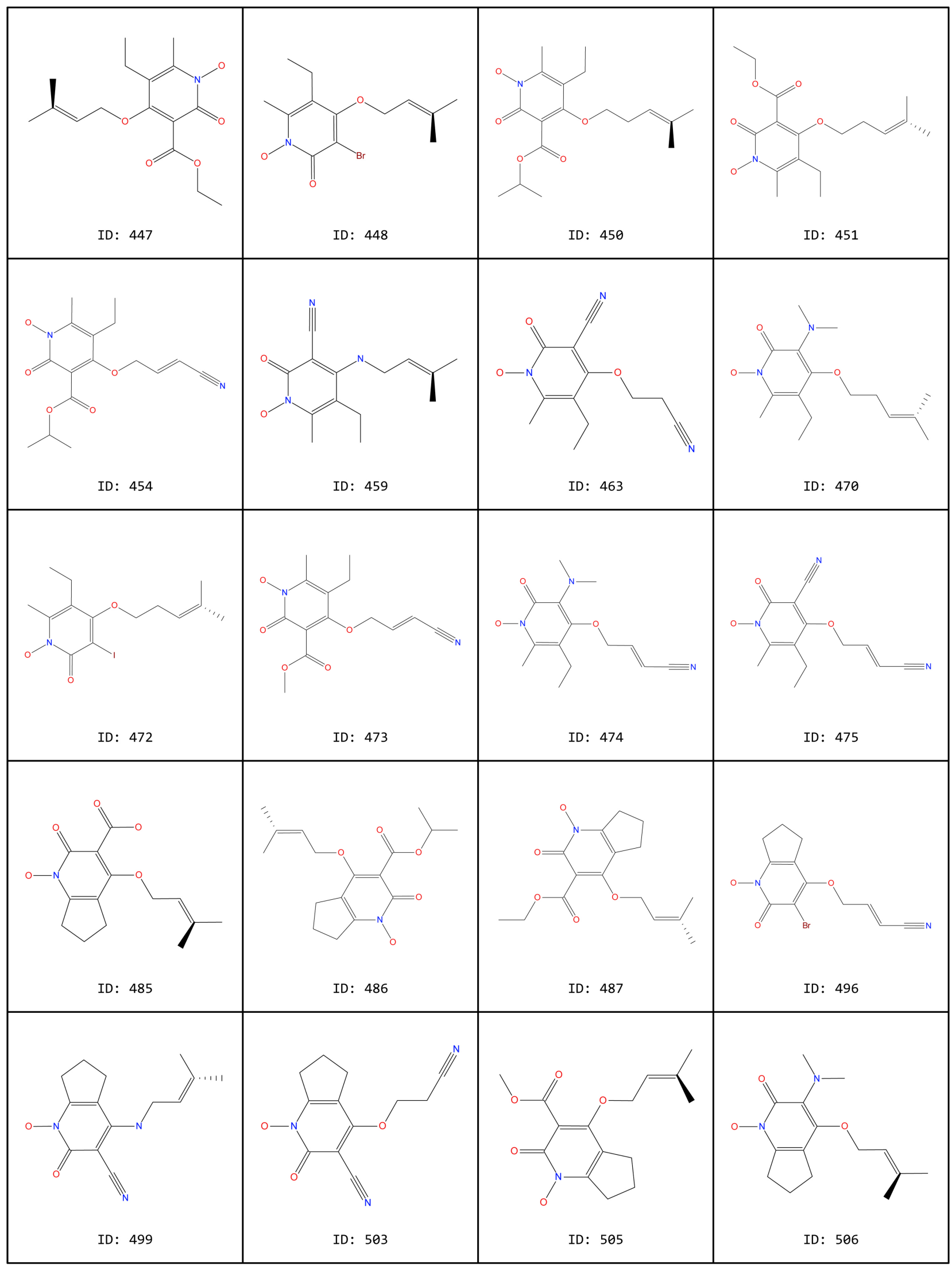




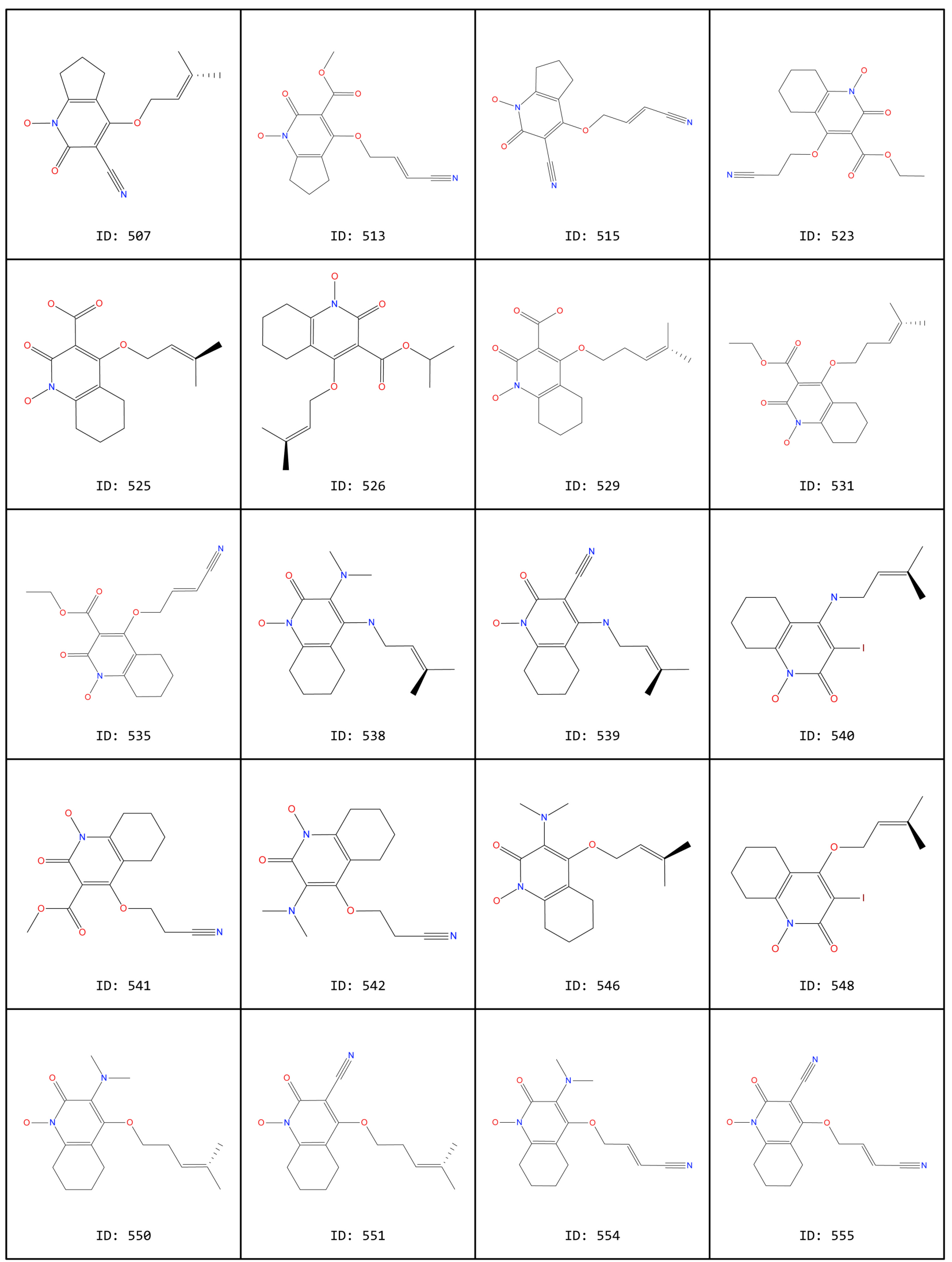

Figure S7. 160 Structures docked with RT. 